\title{
Global Dynamics of Delayed Sigmoid Beverton-Holt Equation
}

\author{
Toufik Khyat and M. R. S. Kulenović \\ Department of Mathematics, University of Rhode Island, Kingston, Rhode Island 02881-0816, USA
}

Correspondence should be addressed to M. R. S. Kulenović; mkulenovic@mail.uri.edu

Received 9 March 2020; Accepted 16 April 2020; Published 26 May 2020

Guest Editor: Abdul Qadeer Khan

Copyright (c) 2020 Toufik Khyat and M. R. S. Kulenović. This is an open access article distributed under the Creative Commons Attribution License, which permits unrestricted use, distribution, and reproduction in any medium, provided the original work is properly cited.

In this paper, certain dynamic scenarios for general competitive maps in the plane are presented and applied to some cases of second-order difference equation $x_{n+1}=f\left(x_{n}, x_{n-1}\right), n=0,1, \ldots$, where $f$ is decreasing in the variable $x_{n}$ and increasing in the variable $x_{n-1}$. As a case study, we use the difference equation $x_{n+1}=\left(x_{n-1}^{2} /\left(c x_{n-1}^{2}+d x_{n}+f\right)\right), n=0,1, \ldots$, where the initial conditions $x_{-1}, x_{0} \geq 0$ and the parameters satisfy $c, d, f>0$. In this special case, we characterize completely the global dynamics of this equation by finding the basins of attraction of its equilibria and periodic solutions. We describe the global dynamics as a sequence of global transcritical or period-doubling bifurcations.

\section{Introduction and Preliminaries}

Consider the following difference equation:

$$
x_{n+1}=\frac{x_{n-1}^{2}}{c x_{n-1}^{2}+d x_{n}+f}, \quad n=0,1, \ldots,
$$

where the initial conditions $x_{-1}, x_{0} \geq 0$ and the parameters satisfy that $c, f \geq 0, d>0$. Equation (1) is a special case of the equation

$$
x_{n+1}=\frac{C x_{n-1}^{2}+D x_{n}+F}{c x_{n-1}^{2}+d x_{n}+f}, \quad n=0,1, \ldots,
$$

where $x_{-1}, x_{0} \geq 0$ and the parameters satisfy $C, D, F, c, d, f \geq 0, \quad C+D+F>0, \quad c+d+f>0, c+D>0$, and $C+d>0$. By using the theory of competitive systems, we will describe precisely the basins of attraction of the equilibrium points and period-two solutions of equation (1).

Both equations (1) and (2) are special cases of

$$
\begin{array}{r}
x_{n+1}=\frac{A x_{n}^{2}+B x_{n} x_{n-1}+C x_{n-1}^{2}+D x_{n}+E x_{n-1}+F}{a x_{n}^{2}+b x_{n} x_{n-1}+c x_{n-1}^{2}+d x_{n}+e x_{n-1}+f}, \\
n=0,1, \ldots,
\end{array}
$$

where all parameters are nonnegative numbers and the initial conditions $x_{-1}, x_{0} \geq 0$ so that the solution is well defined. Many special cases of equation (3) have been investigated in [1-6]. In particular, a special case where $A=$ $C=D=a=c=d=0$ was studied in $[1,2]$, where a variety of techniques was used to obtain some global results. Other special cases of equation (3),

$$
\begin{aligned}
& x_{n+1}=\frac{x_{n-1}^{2}}{a x_{n}^{2}+b x_{n} x_{n-1}+c x_{n-1}^{2}}, \quad n=0,1, \ldots, \\
& x_{n+1}=\frac{x_{n-1}^{2}}{a x_{n}^{2}+c x_{n-1}^{2}+f}, \quad n=0,1, \ldots, \\
& x_{n+1}=\frac{x_{n-1}^{2}}{b x_{n} x_{n-1}+c x_{n-1}^{2}+f}, \quad n=0,1, \ldots,
\end{aligned}
$$

were considered in $[4,7,8]$, respectively, where the theory of monotone maps in the plane was used to derive the global dynamics of these equations. Indeed, in [4], the coexistence of a unique locally asymptotically stable equilibrium point and a locally asymptotically stable minimal period-two solution was obtained for the first time. Equation (1), on the other hand, can have up to three fixed points and up to three 
period-two solutions and its dynamics is similar to the dynamics of equations (5) and (6). The possible dynamic scenarios for equation (1) will be a motivation for getting corresponding results for the general second-order difference equation in Section 2, and these general results will also imply global dynamics results for equations (4)-(6). Thus, we will obtain some general global dynamic results for general second-order difference equation,

$$
x_{n+1}=f\left(x_{n}, x_{n-1}\right), \quad x_{-1}, x_{0} \in I, n=0,1, \ldots,
$$

which will unify global dynamic results for equations (4)-(6) in the hyperbolic case. The nonhyperbolic cases may be different for different equations and require the specific investigation. $d=0$,

Equation (1) contains an interesting special case when

$$
x_{n+1}=\frac{x_{n-1}^{2}}{c x_{n-1}^{2}+f}, \quad n=0,1, \ldots,
$$

which is a well-known delayed sigmoid Beverton-Holt equation of which interesting dynamics is given in [8]. Thus, equation (1) can be considered as a perturbation of equation (8). Some interesting results about boundedness, stability, and attractivity for related ordinary differential equations with or without delay can be found in $[9,10]$.

The organization of the paper is as follows. The rest of this section contains some necessary notions of competitive systems in the plane. Section 2 contains several global dynamic scenarios for two-dimensional competitive systems and maps in the absence of minimal period-two solutions and the applications of these results to a general secondorder difference equation with certain monotonic properties of transition function. Finally, Section 3 gives global dynamic results for equation (1). As we will show, the global dynamics of equation (1) can be described as a sequence of global period-doubling bifurcations with two parameters $d$ and $4 f c$. Three regions for parameter $d$ are $(0,1 / 3),[1 / 3,1)$, and $[1, \infty)$. In the critical region $d \in(0,1 / 3)$, the critical subregions for the bifurcation parameter $4 f c$ are $\left(0,(d-1)^{2}-4 d^{2}\right),\left((d-1)^{2}-4 d^{2},(d-1)^{2}\right),\left((d-1)^{2}, 1\right)$ and $(1, \infty)$. In the critical region $d \in[1 / 3,1)$, the critical subregions for the bifurcation parameter $4 f c$ are $\left(0,(d-1)^{2}\right),\left((d-1)^{2}, 1\right)$ and $(1, \infty)$. In the critical region $d \in[1, \infty)$, the critical subregions for the bifurcation parameter $4 f c$ are $(0,1)$ and $(1, \infty)$ (see Figures $1-3)$. The dynamics of equation (1) is different from the dynamics of equation (6) which is another perturbation of equation (8), considered in [8]. The perturbation term in the case of equation (1) is $d x_{n}$ (depends on population size at $n$-th generation only) while in the case of equation (6) is $b x_{n} x_{n-1}$ (depends on population sizes at $n-1$-th and $n$-th generations) and as expected the number of bifurcations for equation (1) is smaller than the number for equation (6).

A unique new feature of the proofs of presented results is the use of center manifold theory for proving the global stability result in the nonhyperbolic case of equation (1) as well as the use of concavity properties of invariant manifolds in treating some nonhyperbolic cases of equation (1).
There is an extensive literature on the dynamics of monotone maps (competitive and cooperative) that can be found in [11-13]. In this paper, we will use some theorems from [14-16] used in several papers such as $[4-6,8,17,18]$ that will be important in establishing the global dynamics results for equation (1).

We will use Theorem 5 from [19] which states that, for every solution $\left\{x_{n}\right\}_{n=-1}^{\infty}$ of equation (7), the subsequences $\left\{x_{2 n}\right\}_{n=0}^{\infty}$ and $\left\{x_{2 n-1}\right\}_{n=0}^{\infty}$ of even and odd terms are eventually monotonic, provided that a function $f$ is nonincreasing in the first variable and nondecreasing in the second variable. In view of Theorem 5 from [19], determining the basins of attraction of an equilibrium, or a period-two solution or of the point on the boundary where equation (7) may not be defined, becomes major objective of the study of global dynamics.

Remark 1 . The connection between the theory of monotone maps and the asymptotic behavior of equation (7) is the consequence of the fact that if $f$ is strongly decreasing in the first argument and strongly increasing in the second argument, then the second iterate of a map associated to equation (7) is a strictly competitive map on $I \times I$, see [15] and Remarks 2 and 3 in [3].

The fixed point $(\bar{x}, \bar{y})$ of a planar competitive or cooperative map $T$ is said to be nonhyperbolic if the Jacobian matrix has at least one eigenvalue on the unit circle $(|\lambda|=1)$, that is, if $\lambda= \pm 1$. If one eigenvalue is inside the unit circle $(|\lambda|<1)$, the fixed point is nonhyperbolic of stable type, and if the other eigenvalue is outside of the unit circle $(|\lambda|>1)$, the fixed point is nonhyperbolic of unstable type. If both eigenvalues lie on the unit circle, the fixed point is nonhyperbolic of resonance type of either $(1,1),(1,-1),(-1,1)$, or $(-1,-1)$ depending on the values of the eigenvalues. It should be noticed that the eigenvalues of the Jacobian matrix of planar competitive or cooperative map are real numbers with a largest eigenvalue which is positive.

\section{Main Results}

We start with specific global dynamic scenarios for competitive system (9) that will be applied to equation (7).

Theorem 1. Considering the competitive map T generated by the system,

$$
\left\{\begin{array}{l}
x_{n=1}=f\left(x_{n}, y_{n}\right), \\
y_{n+1}=g\left(x_{n}, y_{n}\right), \\
n=0,1, \ldots,\left(x_{-1}, x_{0}\right) \in R
\end{array}\right.
$$

on a set $\mathscr{R}$ with a nonempty interior.

(a) Assume that $T$ has seven fixed points $E_{1}, \ldots, E_{7}$ such that five belongs to the west and south boundaries of the region $\mathscr{R}$ and two fixed points are interior points. Moreover, assuming that $E_{1}$ and $E_{2}$ belong to the west boundary, $E_{3}$ is the south-west corner of the region $\mathscr{R}$, and $E_{4}$ and $E_{5}$ are on the south boundary of $\mathscr{R}$ such that $E_{1} \preceq_{s e} E_{2} \preceq_{s e} E_{3} \varsigma_{s e} E_{4}{ }_{s e} E_{5}$. Moreover, assume 


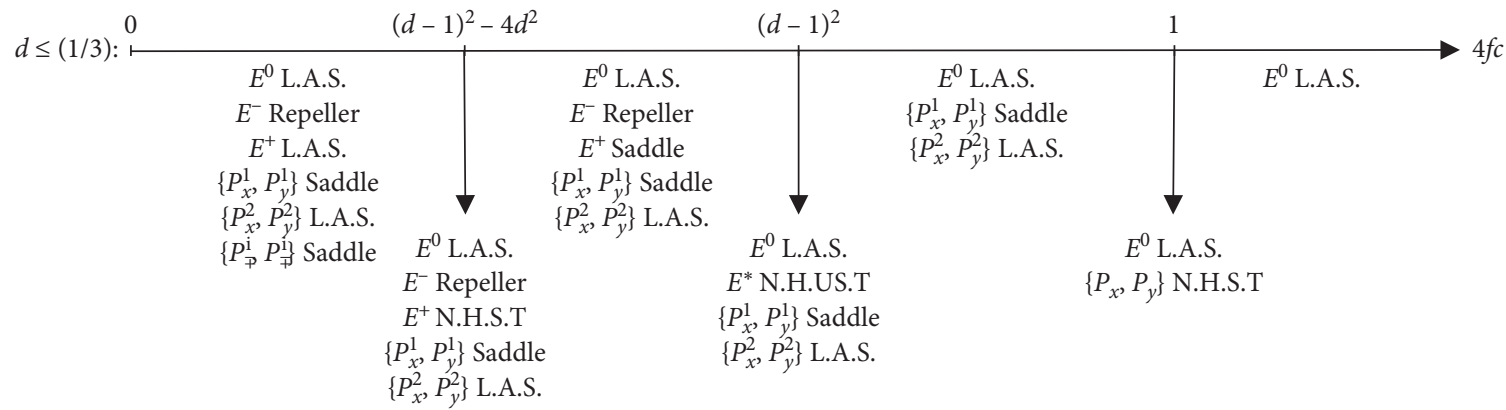

FIgURe 1: Bifurcation diagram of global dynamics of equation (1) in the parametric region $d<1 / 3$ for different values of the bifurcation parameter $4 f c$.

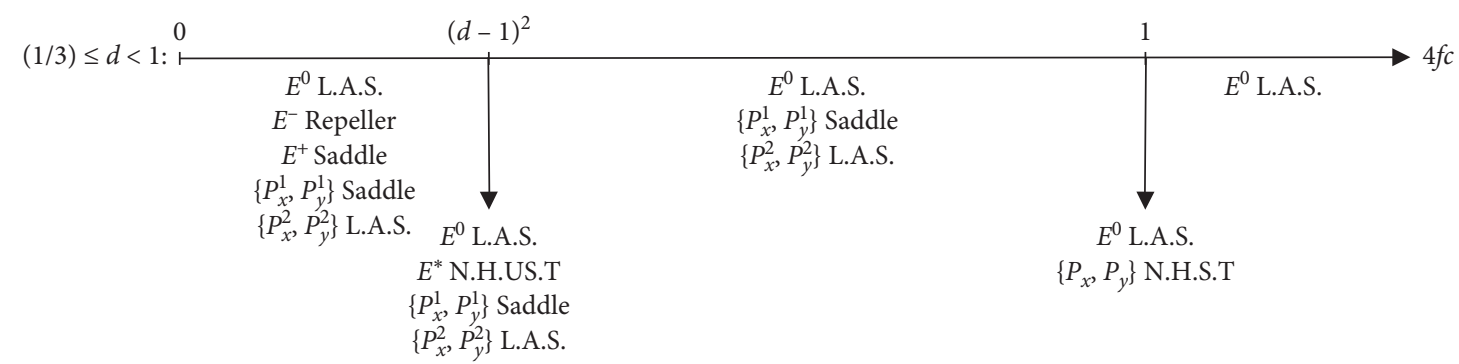

Figure 2: Bifurcation diagram of global dynamics of equation (1) in the parametric region $1 / 3 \leq d<1$ for different values of the bifurcation parameter $4 f c$.

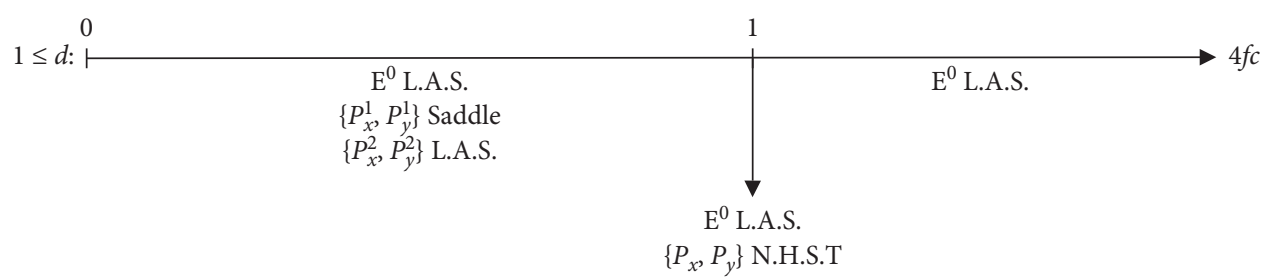

FIGURE 3: Bifurcation diagram of global dynamics of equation (1) in the parametric region $1 \leq d$ for different values of the bifurcation parameter $4 f c$.

that $E_{6} \preceq_{n e} E_{7}$ and that $E_{6} \notin\left[E_{2}, E_{4}\right]$ and $E_{7} \in\left[E_{1}, E_{5}\right]$. Finally, assume that $E_{1}, E_{3}, E_{5}$ are locally asymptotically stable, $E_{6}$ is a repeller, and $E_{2}, E_{4}$ are saddle points. If $E_{7}$ is either a saddle point or a nonhyperbolic point of stable type and $T$ has no period-two solutions, then all solutions which start between the stable manifolds $\mathscr{W}^{s}\left(E_{2}\right)$ and $\mathscr{W}^{s}\left(E_{4}\right)$ converge to $E_{3}$ and all solutions which start between the stable manifolds $\mathscr{W}^{s}\left(E_{2}\right)$ and $\mathscr{W}^{s}\left(E_{7}\right)$ converge to $E_{1}$ and all solutions which start between the stable manifolds $\mathscr{W}^{s}\left(E_{4}\right)$ and $\mathscr{W}^{s}\left(E_{7}\right)$ converge to $E_{5}$.

(b) Assume that $T$ has exactly six fixed points $E_{1}, \ldots, E_{6}$, where the points have the same configuration and points $E_{1}, \ldots, E_{5}$ have the same local character as in part (a), while $E_{6}$ is a nonhyperbolic point of unstable type. If $T$ has no period-two solutions, then there exist two increasing continuous curves $C_{1}$ and $C_{2}, C_{2} \varsigma_{s e} C_{1}$ emanating from $E_{6}$ such that all solutions which start between $C_{1}$ and $C_{2}$ converge to $E_{6}$. Furthermore, all solutions which start between the stable manifolds $\mathscr{W}^{s}\left(E_{2}\right)$ and $\mathscr{W}^{s}\left(E_{4}\right)$ converge to $E_{1}$, and all solutions which start above $\mathscr{W}^{s}\left(E_{2}\right) \cup C_{2}$ converge to $E_{1}$ and all solutions which start below $\mathscr{W}^{s}\left(E_{4}\right) \cup C_{1}$ converge to $E_{5}$.

(c) Assume that $T$ has exactly nine fixed points $E_{1}, \ldots, E_{9}$, where the points $E_{1}, \ldots, E_{7}$ have the same configuration and the same local character as in (a). Assuming that the fixed points $E_{8}, E_{9}$ are saddle points such that $E_{8} \in\left[E_{1}, E_{7}\right]$ and $E_{9} \in\left[E_{7}, E_{5}\right]$. Assume that $T$ has no period-two solutions, then all solutions which start between the stable manifolds $\mathscr{W}^{s}\left(E_{2}\right)$ and $\mathscr{W}^{s}\left(E_{4}\right)$ converge to $E_{3}$, all solutions which start above $\mathscr{W}^{s}\left(E_{2}\right) \cup \mathscr{W}^{s}\left(E_{8}\right)$ converges to $E_{1}$, and all solutions which start below $\mathscr{W}^{s}\left(E_{4}\right) \cup \mathscr{W}^{s}\left(E_{9}\right)$ converge to $E_{5}$. 
Finally, all solutions which start between the stable manifolds $\mathscr{W}^{s}\left(E_{8}\right)$ and $\mathscr{W}^{s}\left(E_{9}\right)$ converge to $E_{7}$.

Proof

(a) The existence of the global stable and unstable manifolds of the saddle fixed points is guaranteed by Theorems $1-5$ in [15]. In any case, all global stable manifolds $\mathscr{W}^{s}\left(E_{2}\right), \mathscr{W}^{s}\left(E_{4}\right)$ and $\mathscr{W}^{s}\left(E_{7}\right)$ have an endpoint at $E_{6}$, and $\mathscr{W}^{s}\left(E_{7}\right)$ has another endpoint at $(\infty, \infty)$.

In view of Theorem 4 in [15], every solution which starts between the stable manifolds $\mathscr{W}^{s}\left(E_{2}\right)$ and $\mathscr{W}^{s}\left(E_{4}\right)$ eventually enters int $\left[E_{2}, E_{4}\right]$. If the initial point $\left(x_{0}, y_{0}\right)$ is in $\operatorname{int}\left[E_{2}, E_{4}\right]$, one can find the points $\left(x_{l}, y_{l}\right)$ on the $y$-axis and $\left(x_{u}, y_{u}\right)$ on the $x$-axis such that $\left(x_{l}, y_{l}\right) \preceq_{s e}\left(x_{0}, y_{0}\right) \preceq_{s e}\left(x_{u}, y_{u}\right)$. This will imply that $T^{n}\left(\left(x_{l}, y_{l}\right)\right) \preceq_{s e} T^{n}\left(\left(x_{0}, y_{0}\right)\right)$ $\preceq_{s e} T^{n}\left(\left(x_{u}, y_{u}\right)\right)$, and so $T^{n}\left(\left(x_{0}, y_{0}\right)\right)$ converges to $E_{3}$ as both $T^{n}\left(\left(x_{l}, y_{l}\right)\right), T^{n}\left(\left(x_{u}, y_{u}\right)\right)$ do.

If the initial point $\left(x_{0}, y_{0}\right)$ is above $\mathscr{W}^{s}\left(E_{2}\right) \cup \mathscr{W}^{s}\left(E_{7}\right)$, one can find the point $\left(x_{l}, y_{l}\right)$ on the $y$-axis and a point $\left(x_{u}, y_{u}\right) \in \mathscr{W}^{s}\left(E_{2}\right) \cup \mathscr{W}^{s}\left(E_{7}\right)$ such that $\left(x_{l}, y_{l}\right) \preceq_{s e}\left(x_{0}, y_{0}\right) \preceq_{s e}\left(x_{u}, y_{u}\right)$. This will imply that $T^{n}\left(\left(x_{l}, y_{l}\right)\right) \preceq_{s e} T^{n}\left(\left(x_{0}, y_{0}\right)\right) \preceq_{s e} T^{n}\left(\left(x_{u}\right.\right.$, $\left.\left.y_{u}\right)\right)$, and so $T^{n}\left(\left(x_{0}, y_{0}\right)\right) \in \operatorname{int}\left[E_{1}, E_{7}\right]$ eventually. Now, in view of Corollary 1 in [15], $T^{n}\left(\left(x_{0}, y_{0}\right)\right) \longrightarrow E_{1}$ as $n \longrightarrow \infty$.

In a similar way the case when the initial point $\left(x_{0}, y_{0}\right)$ is below $\mathscr{W}^{s}\left(E_{4}\right) \cup \mathscr{W}^{s}\left(E_{7}\right)$ can be handled.

(b) The existence of the global stable and unstable manifolds of the saddle fixed points is guaranteed by Theorems $1-5$ in [15]. Both global stable manifolds $\mathscr{W}^{s}\left(E_{2}\right)$ and $\mathscr{W}^{s}\left(E_{4}\right)$ have an endpoint at $E_{6}$. The existence of curves $C_{1}$ and $C_{2}$ follows from Theorem 2 in [16]. The proof that the region between the stable manifolds $\mathscr{W}^{s}\left(E_{2}\right)$ and $\mathscr{W}^{s}\left(E_{4}\right)$ eventually enters $\operatorname{int}\left[E_{2}, E_{4}\right]$ and so it converges to $E_{3}$ is the same as in part (a).

In a similar way as in the proof of part (a), we can show that if the initial point $\left(x_{0}, y_{0}\right)$ is above $\mathscr{W}^{s}\left(E_{2}\right) \cup C_{2}$ it will eventually enter int $\left[E_{1}, E_{6}\right]$ and so it will converge to $E_{1}$. In a similar way, we can show that if the initial point $\left(x_{0}, y_{0}\right)$ is below $\mathscr{W}^{s}\left(E_{4}\right) \cup C_{1}$, it will eventually enter int $\left[E_{6}, E_{5}\right]$ and so it will converge to $E_{5}$.

Finally, if the initial point $\left(x_{0}, y_{0}\right)$ is between $C_{1}$ and $C_{2}$, then one can find the point $\left(x_{l}, y_{l}\right) \in C_{2}$ and a point $\left(x_{u}, y_{u}\right) \in C_{1}$ such that $\left(x_{l}, y_{l}\right) \preceq_{s e}\left(x_{0}, y_{0}\right)$ $\preceq_{s e}\left(x_{u}, y_{u}\right)$. This will imply that $T^{n}\left(\left(x_{l}, y_{l}\right)\right) \preceq_{s e} T^{n}$ $\left(\left(x_{0}, y_{0}\right)\right) \preceq_{s e} T^{n}\left(\left(x_{u}, y_{u}\right)\right)$, and so $T^{n}\left(\left(x_{0}, y_{0}\right)\right) \longrightarrow$ $E_{6}$ as $T^{n}\left(\left(x_{u}, y_{u}\right)\right) \longrightarrow E_{6}, T^{n}\left(\left(x_{l}, y_{l}\right)\right) \longrightarrow E_{6}$.

(c) The proof that the region between the stable manifolds $\mathscr{W}^{s}\left(E_{2}\right)$ and $\mathscr{W}^{s}\left(E_{4}\right)$ is the basin of attraction of $E_{3}$ is same as in part (a) and will be omitted. The proof that all solutions which start above $\mathscr{W}^{s}\left(E_{2}\right) \cup \mathscr{W}^{s}\left(E_{8}\right)$ converges to $E_{1}$ and all solutions which start below $\mathscr{W}^{s}\left(E_{4}\right) \cup \mathscr{W}^{s}\left(E_{9}\right)$ converge to $E_{5}$ is same as in part (a) and so will be omitted.

If the initial point $\left(x_{0}, y_{0}\right)$ is between $\mathscr{W}^{s}\left(E_{8}\right)$ and $\mathscr{W}^{s}\left(E_{9}\right)$, then one can find the point $\left(x_{l}, y_{l}\right) \in$ $\mathscr{W}^{s}\left(E_{8}\right)$ and a point $\left(x_{u}, y_{u}\right) \in \mathscr{W}^{s}\left(E_{9}\right)$ such that $\left(x_{l}, y_{l}\right) \preceq_{s e}\left(x_{0}, y_{0}\right) \preceq_{s e}\left(x_{u}, y_{u}\right)$. This will imply that $T^{n}\left(\left(x_{l}, y_{l}\right)\right) \preceq_{s e} T^{n}\left(\left(x_{0}, y_{0}\right)\right) \preceq_{s e} T^{n}\left(\left(x_{u}, y_{u}\right)\right)$, and so $T^{n}\left(\left(x_{0}, y_{0}\right)\right) \in \operatorname{int}\left[E_{8}, E_{9}\right]$ as $T^{n}\left(\left(x_{u}, y_{u}\right)\right) \longrightarrow E_{9}, T^{n}$ $\left(\left(x_{l}, y_{l}\right)\right) \longrightarrow E_{8}$ as $n \longrightarrow \infty$. Now, in view of Corollary 2 in [14], $T^{n}\left(\left(x_{0}, y_{0}\right)\right) \longrightarrow E_{7}$ as $n \longrightarrow \infty$.

Theorem 2. Consider equation (7) and assume that $f$ is decreasing in the first and increasing in the second variable on the set $(a, b)^{2}$.

(a) Assume that equation (7) has three equilibrium points $E_{0} \preceq_{n e} E^{-} \preceq_{n e} E^{+}$, where $E_{0}$ is locally asymptotically stable, $E^{-}$is a repeller, and $E^{+}$is either a saddle point or a nonhyperbolic point of stable type. Furthermore, assume that equation (7) has two minimal period-two solutions $\left\{P_{1}, Q_{1}\right\},\left\{P_{2}, Q_{2}\right\}$ such that $P_{2} \preceq_{s e} P_{1} \preceq_{s e} E_{0}$ $\preceq_{s e} Q_{1}{ }_{s e} Q_{2}$ and $E^{-}, E^{+} \in\left[P_{2}, Q_{2}\right] \backslash\left[P_{1}, Q_{1}\right]$. If $\left\{P_{1}, Q_{1}\right\}$ is a saddle point and $\left\{P_{2}, Q_{2}\right\}$ is locally asymptotically stable, then every solution with the initial point between the stable manifolds $\mathscr{W}^{s}\left(P_{1}\right)$ and $\mathscr{W}^{s}\left(Q_{1}\right)$ converges to $E_{0}$ while every solution which starts off $\mathscr{W}^{s}\left(P_{1}\right) \cup \mathscr{W}^{s}\left(Q_{1}\right) \cup \mathscr{W}^{s}\left(E^{+}\right)$converges to the periodic solution $\left\{P_{2}, Q_{2}\right\}$.

(b) Assume that equation (7) has two equilibrium points $E_{0} \preceq_{n e} E$, where $E_{0}$ is locally asymptotically stable and $E^{+}$is a nonhyperbolic point of unstable type. Furthermore, assume that equation (7) has two minimal period-two solutions $\left\{P_{1}, Q_{1}\right\},\left\{P_{2}, Q_{2}\right\}$ such that $P_{2} \varsigma_{s e} P_{1}{ }_{s e} E_{0} \varsigma_{s e} Q_{1} \preceq{ }_{s e} Q_{2}$ and $E^{-}, E^{+} \in\left[P_{2}, Q_{2}\right] \backslash$ $\left[P_{1}, Q_{1}\right]$. If $\left\{P_{1}, Q_{1}\right\}$ is a saddle point and $\left\{P_{2}, Q_{2}\right\}$ is locally asymptotically stable, then every solution which starts between the stable manifolds $\mathscr{W}^{s}\left(P_{1}\right)$ and $\mathscr{W}^{s}\left(Q_{1}\right)$ converges to $E_{0}$ while every solution which starts above $\mathscr{W}^{s}\left(P_{1}\right) \cup C_{2}$ and below $\mathscr{W}^{s}\left(Q_{1}\right) \cup C_{1}$ converges to the periodic solution $\left\{P_{2}, Q_{2}\right\}$. Finally, every solution starting between $C_{1}$ and $C_{2}$ converges to $E$.

(c) Assume that equation (7) has three equilibrium points $E_{0} \preceq_{n e} E^{-} \preceq_{n e} E^{+}$, where $E_{0}$ and $E^{+}$are locally asymptotically stable and $E^{-}$is a repeller. Furthermore, assume that equation (7) has three minimal periodtwo solutions $\left\{P_{1}, Q_{1}\right\},\left\{P_{2}, Q_{2}\right\},\left\{P_{3}, Q_{3}\right\}$ such that $P_{2} \unlhd_{s e} P_{1} \preceq{ }_{s e} E_{0}{ }_{s e} Q_{1} \preceq{ }_{s e} Q_{2}$ and $E^{-}, E^{+} \in\left[P_{2}, Q_{2}\right] \backslash$ $\left[P_{1}, Q_{1}\right]$ and $P_{2} \preceq_{s e} P_{3} \preceq_{s e} E^{+} \preceq_{s e} Q_{3} \preceq_{s e} Q_{2}$.

If $\left\{P_{1}, Q_{1}\right\}$ and $\left\{P_{3}, Q_{3}\right\}$ are saddle points and $\left\{P_{2}, Q_{2}\right\}$ is locally asymptotically stable, then every solution which starts between the stable manifolds $\mathscr{W}^{s}\left(P_{1}\right)$ and $\mathscr{W}^{s}\left(Q_{1}\right)$ converges to $E_{0}$ and every solution which starts between the stable manifolds $\mathscr{W}^{s}\left(P_{3}\right)$ and $\mathscr{W}^{s}\left(Q_{3}\right)$ converges to $E_{7}$ while every solution 
which starts off in the complement of the basins of attractions of $E_{0}, E_{7},\left\{P_{1}, Q_{1}\right\}$ and $\left\{P_{3}, Q_{3}\right\}$ converges to the periodic solution $\left\{P_{2}, Q_{2}\right\}$.

Proof. In view of Remark 1 and Theorem 5 from [19], the second iterate $T^{2}$ of the map $T$ associated with equation (7) is strictly competitive and does not have any period-two points.

(a) By noticing that the period-two points of $T$ are the fixed points of $T^{2}$, two period-two solutions $\left\{P_{1}, Q_{1}\right\},\left\{P_{2}, Q_{2}\right\}$ become four fixed points of $T^{2}$. An application of Theorem 1 in part (a) to $T^{2}$ completes the proof.

(b) In view of Remark 1 , the second iterate $T^{2}$ of the map $T$ associated with equation (7) is strictly competitive and has six equilibrium points $E_{1}=P_{2}, E_{2}=P_{1}$, $E_{3}=E_{0}, E_{4}=Q, E_{5}=Q_{2}$ and $E_{6}=E$. An application of Theorem 1 in part (b) to $T^{2}$ yields $\lim _{n \longrightarrow \infty} T^{2 n}$ $\left(\left(x_{0}, y_{0}\right)\right)=E_{0}$ for every $\left(x_{0}, y_{0}\right)$ between the stable manifolds $\mathscr{W}^{s}\left(P_{1}\right)$ and $\mathscr{W}^{s}\left(Q_{1}\right)$. Furthermore, we derive

$$
\lim _{n \longrightarrow \infty} T^{2 n+1}\left(\left(x_{0}, y_{0}\right)\right)=\lim _{n \longrightarrow \infty} T\left(T^{2 n}\left(\left(x_{0}, y_{0}\right)\right)\right)=T\left(\lim _{n \longrightarrow \infty} T^{2 n}\left(\left(x_{0}, y_{0}\right)\right)\right)=T\left(E_{2}\right)=E_{2}
$$

where we used a continuity of the map $T$. Consequently, $\lim _{n \rightarrow \infty} T^{n}\left(\left(x_{0}, y_{0}\right)\right)=E_{0}$. The proof of other cases is similar.

(c) By noticing that the period-two points of $T$ are the fixed points of $T^{2}$, three period-two solutions $\left\{P_{1}, Q_{1}\right\},\left\{P_{2}, Q_{2}\right\},\left\{P_{3}, Q_{3}\right\}$ become six fixed points of $T^{2}$. Applying Theorem 1 in part (c) to $T^{2}$, we complete the proof.

\section{Case Study: Equation (1)}

3.1. Local Stability Analysis for Equilibria. An equilibrium solution of equation (1) must satisfy

$$
\bar{x}=\frac{\bar{x}^{2}}{c \bar{x}^{2}+d \bar{x}+f},
$$

i.e., $\bar{x}=0$ or $c \bar{x}^{2}+(d-1) \bar{x}+f=0$. Therefore, equation (1) has the following:

(1) The unique equilibrium $E_{0}=(0,0)$, if $d \geq 1$ or $(d<1$ and $\left.(d-1)^{2}-4 f c<0\right)$

(2) Two equilibrium points $E_{0}$ and $E^{*}=(((1-d) / 2 c)$, $((1-d) /(2 c)))$, if $d<1$ and $(d-1)^{2}-4 f c=0$

(3) Three equilibrium points $E_{0}$ and $E_{ \pm}=(((1-d) \pm$ $\left.\sqrt{(1-d)^{2}-4 f c} / 2 c\right),\left(\left((1-d) \pm \sqrt{\left.(1-d)^{2}-4 f c\right)}\right)\right.$ 2c))otherwise

Denote $F(u, v)=v^{2} /\left(c v^{2}+d u+f\right)$; then equation (1) has the following linearized equation:

$$
z_{n+1}=p z_{n}+q z_{n-1}
$$

where

$$
\begin{aligned}
& p=\frac{\partial F}{\partial u}(\bar{x}, \bar{x})=\frac{d \bar{x}^{2}}{\left(c \bar{x}^{2}+d \bar{x}+f\right)^{2}} \\
& q=\frac{\partial F}{\partial v}(\bar{x}, \bar{x})=\frac{2 \bar{x}(d \bar{x}+f)}{\left(c \bar{x}^{2}+d \bar{x}+f\right)^{2}} .
\end{aligned}
$$

If $\bar{x}=0$, then clearly $p=q=0$. If $\bar{x} \neq 0$, then by the equilibrium equation,

$$
\begin{aligned}
& p=-d, \\
& q=2\left(d+\frac{f}{\bar{x}}\right)=2(1-c \bar{x}) .
\end{aligned}
$$

Proposition 1. Given that $c, d, f>0$,

(1) the equilibrium $E_{0}$ is locally asymptotically stable for all values of the parameter.

(2) If $d<1$ and $(d-1)^{2}-4 f c=0$, then the positive equilibrium point $E^{*}$ is nonhyperbolic of unstable type.

(3) If $d<1$ and $(d-1)^{2}-4 f c>0$, then the equilibrium point $E^{-}$is a repeller while the stability of $E^{+}$is subject to the following conditions:

(a) $E^{+}$is locally asymptotically stable if $(d-1)^{2}-$ $4 f c>4 d^{2}$

(b) $E^{+}$is nonhyperbolic of stable type if $(d-1)^{2}-$ $4 f c=4 d^{2}$

(c) $E^{+}$is a saddle point if $(d-1)^{2}-4 f c<4 d^{2}$

Proof

(1) Since $p=q=0$ for $\bar{x}=0$ implies that the unique eigenvalue $\lambda=0, E_{0}$ is locally asymptotically stable for all values of $c, d$, and $f$.

(2) As $p=-d, q=2(1-c \bar{x})$ and $\bar{x}^{*}=(1-d) / 2 c$, then the characteristic equation is given by

$$
\lambda^{2}+d \lambda-(d+1)=0
$$

which solutions are $\lambda_{+}=1$ and $\lambda_{-}=-(d+1)$. The latter shows that $E^{*}$ is nonhyperbolic of unstable type. 
(3) The roots of the characteristic equation are $\lambda_{+}=\left(-d+\sqrt{d^{2}+8(1-c \bar{x})}\right) / 2>0$ and $\lambda_{-}=(-d-$ $\left.\sqrt{d^{2}+8(1-c \bar{x})}\right) / 2<0$.

For $E^{-}$,

since $\bar{x}_{-}<(1-d) / 2 c$, one can easily check that $\sqrt{d^{2}+8\left(1-c \bar{x}_{-}\right)}>(2+d)$ which implies $\lambda_{+}>1$.

On the other hand, one can use the fact that $\bar{x}_{-}<(1+$ d) $/ 2 c$ to show that $\lambda_{-}<-1$.

For $E^{+}$,

since $\bar{x}_{+}>(1-d) / 2 c$, one can similarly show that $\lambda_{+}<1$.

Moreover, a simple algebraic verification shows the following:

(i) $\left|\lambda_{-}\right|<1$ for $4 d^{2}<(1-d)^{2}-4 f c$;

(ii) $\left|\lambda_{-}\right|=1$ for $4 d^{2}=(1-d)^{2}-4 f c$;

(iii) $\left|\lambda_{-}\right|>1$ for $4 d^{2}>(1-d)^{2}-4 f c$.

Consequently, we conclude that $E^{-}$is a repeller whenever it exists while

(i) $E^{+}$is locally asymptotically stable, for $4 d^{2}<$ $(1-d)^{2}-4 f c$;

(ii) $E^{+}$is nonhyperbolic of stable type, for $4 d^{2}=$ $(1-d)^{2}-4 f c$

(iii) $E^{+}$is a saddle point, for $4 d^{2}>(1-d)^{2}-4 f c$.
3.2. Local Stability Analysis of Minimal Period-Two Solutions. Here, we present the results about the existence and stability of minimal period-two solutions of equation (1).

Theorem 3. Given that $c, d, f>0$, we have the following:

(1) If $4 f c>1$, then equation (1) has no minimal periodtwo solutions.

(2) If $4 f c=1$, then equation (1) has a minimal periodtwo solution: $\left\{P_{x}(1 / 2 c, 0), P_{y}(0,1 / 2 c)\right\}$.

(3) If $(d-1)^{2}-4 d^{2} \leq 4 f c<1$, then equation (1) has two minimal period-two solutions:

$$
\begin{aligned}
& \left\{P_{x}^{1}\left(\frac{1-\sqrt{1-4 c f}}{2 c}, 0\right), P_{y}^{1}\left(0, \frac{1-\sqrt{1-4 c f}}{2 c}\right)\right\}, \\
& \left\{P_{x}^{2}\left(\frac{1+\sqrt{1-4 c f}}{2 c}, 0\right), P_{y}^{2}\left(0, \frac{1+\sqrt{1-4 c f}}{2 c}\right)\right\} .
\end{aligned}
$$

(4) If $4 f c<(d-1)^{2}-4 d^{2}$, then equation (1) has three minimal period-two solutions:

$$
\begin{aligned}
& \left\{P_{x}^{1}, P_{y}^{1}\right\}, \\
& \left\{P_{x}^{2}, P_{y}^{2}\right\}, \\
& \left\{P_{\mp}^{i}\left(\frac{1+d-\sqrt{(1-d)^{2}-4 d^{2}-4 f c}}{2 c}, \frac{1+d+\sqrt{(1-d)^{2}-4 d^{2}-4 f c}}{2 c}\right),\right. \\
& \left.P_{ \pm}^{i}\left(\frac{1+d+\sqrt{(1-d)^{2}-4 d^{2}-4 f c}}{2 c}, \frac{1+d-\sqrt{(1-d)^{2}-4 d^{2}-4 f c}}{2 c}\right)\right\} .
\end{aligned}
$$

Proof. Any period-two solution $(\phi, \psi)$ satisfies the following system of equations:

$$
\left\{\begin{array}{l}
\phi=\frac{\phi^{2}}{c \phi^{2}+d \psi+f} \\
\psi=\frac{\psi^{2}}{c \psi^{2}+d \phi+f}
\end{array}\right.
$$

It follows that

If $\psi=0$, the first equation becomes $c \phi^{2}-\phi+f=0$.

If $\phi=0$, then the second equation turns into $c \psi^{2}-\psi+f=0$.

If $\phi \neq 0$ and $\psi \neq 0$, then the system is equivalent to

$$
\left\{\begin{array}{l}
c \phi^{2}+d \psi-\phi+f=0, \\
c \psi^{2}+d \phi-\psi+f=0, \\
\quad \Longrightarrow c\left(\phi^{2}-\psi^{2}\right)+d(\psi-\phi)+(\psi-\phi)=0 .
\end{array}\right.
$$

Now, given that $\phi \neq \psi$, we get $\phi=((1+d) / c)-\psi$ which implies

$$
c \psi^{2}-(1+d) \psi+\frac{1+d}{c}+f=0
$$

Thus, for solution of the form $(0, \psi)$ or $(\phi, 0)$, we must have $1-4 f c \geq 0$ and for solutions of the form $(\phi, \psi), \phi \neq 0$, $\psi \neq 0$, we must have $(1-d)^{2}-4 d^{2}-4 f c>0$. Consequently, 
(1) if $4 f c>1$, then $1-4 f c<0$ and $(1-d)^{2}-4 d^{2}-4 f c$ $<0$. It follows that equation (1) has no minimal period-two solutions.

(2) If $4 f c=1$, it follows that $\left\{P_{x}, P_{y}\right\}$ is the unique minimal period-two solution of equation (1).

(3) If $(1-d)^{2}-4 d^{2} \leq 4 f c<1$, equation (1) has two minimal period-two solutions:

$$
\begin{aligned}
& \left\{P_{x}^{1}\left(\frac{1-\sqrt{1-4 c f}}{2 c}, 0\right), P_{y}^{1}\left(0, \frac{1-\sqrt{1-4 c f}}{2 c}\right)\right\}, \\
& \left\{P_{x}^{2}\left(\frac{1+\sqrt{1-4 c f}}{2 c}, 0\right), P_{y}^{2}\left(0, \frac{1+\sqrt{1-4 c f}}{2 c}\right)\right\} .
\end{aligned}
$$

(4) If $4 f c<(1-d)^{2}-4 d^{2}$, then equation (1) has three minimal period-two solutions:

$\left\{P_{x}^{1}, P_{y}^{1}\right\},\left\{P_{x}^{2}, P_{y}^{2}\right\}$ together with

$$
\begin{aligned}
& \left\{P_{\mp}^{i}=\left(\frac{1+d-\sqrt{(1-d)^{2}-4 d^{2}-4 f c}}{2 c}, \frac{1+d+\sqrt{(1-d)^{2}-4 d^{2}-4 f c}}{2 c}\right)\right\}, \\
& \left\{P_{ \pm}^{i}=\left(\frac{1+d+\sqrt{(1-d)^{2}-4 d^{2}-4 f c}}{2 c}, \frac{1+d-\sqrt{(1-d)^{2}-4 d^{2}-4 f c}}{2 c}\right)\right\} .
\end{aligned}
$$

Now, consider the following substitution, $u_{n}=x_{n-1}$ and $v_{n}=x_{n}$, which transforms equation (1) into the following two-dimensional system,

$$
\left\{\begin{array}{l}
u_{n+1}=v_{n}, \\
v_{n+1}=\frac{u_{n}^{2}}{c u_{n}^{2}+d v_{n}+f},
\end{array}\right.
$$

to which corresponds the following map:

$$
T\left(\begin{array}{l}
u \\
v
\end{array}\right)=\left(\begin{array}{c}
v \\
h(u, v)
\end{array}\right)=\left(\begin{array}{c}
v \\
\frac{u^{2}}{c u^{2}+d v+f}
\end{array}\right)
$$

in which second iterate $T^{2}$ is given by

$$
T^{2}\left(\begin{array}{l}
u \\
v
\end{array}\right)=T\left(\begin{array}{c}
v \\
h(u, v)
\end{array}\right)=\left(\begin{array}{c}
h(u, v) \\
h(v, h(u, v))
\end{array}\right)=\left(\begin{array}{c}
G(u, v) \\
H(u, v)
\end{array}\right),
$$

where

$$
H(u, v)=\frac{v^{2}}{c v^{2}+d h(u, v)+f} .
$$

The map $T^{2}$ is strongly competitive and its Jacobian matrix is given by

$$
J_{T^{2}}(u, v)=\left(\begin{array}{cc}
\frac{2 u(f+d v)}{\left(c u^{2}+f+d v\right)^{2}} & -\frac{d u^{2}}{\left(c u^{2}+f+d v\right)^{2}} \\
-\frac{2 d u v^{2}(f+d v)}{\left(d u^{2}+\left(c u^{2}+f+d v\right)\left(c v^{2}+f\right)\right)^{2}} & \frac{v\left(\left(\left(d\left(2 c u^{2}+2 f+3 d v\right) u^{2}\right) /\left(c u^{2}+f+d v\right)^{2}\right)+2 f\right)}{\left(\left(d u^{2} /\left(c u^{2}+f+d v\right)\right)+c v^{2}+f\right)^{2}}
\end{array}\right) .
$$

The following theorem describes the local stability of minimal period-two solutions of equation (1) whenever they exist.

\section{Theorem 4}

(1) The minimal period-two solutions $\left\{P_{x}, P_{y}\right\}$ are nonhyperbolic of stable type. 
(2) The minimal period-two solutions $\left\{P_{x}^{1}, P_{y}^{1}\right\}$ are saddle points while $\left\{P_{x}^{2}, P_{y}^{2}\right\}$ are locally asymptotically stable.

(3) The minimal period-two solutions $\left\{P_{\mp}^{i}, P_{ \pm}^{i}\right\}$ are saddle points.

Proof

(1) The minimal period solutions $\left\{P_{x}, P_{y}\right\}$ exist when $4 f c=1$; thus, the Jacobian matrix of the second iterate of the map $T$ at $P_{x}$ and $P_{y}$ is the following:

$$
\begin{aligned}
& J_{T^{2}}\left(P_{x}\right)=\left(\begin{array}{cc}
1 & -d \\
0 & 0
\end{array}\right), \\
& J_{T^{2}}\left(P_{y}\right)=\left(\begin{array}{ll}
0 & 0 \\
0 & 1
\end{array}\right),
\end{aligned}
$$

with the eigenvalues $\lambda_{1}=0$ and $\lambda_{2}=1$; therefore, $\left\{P_{x}, P_{y}\right\}$ are nonhyperbolic of stable type.

(2) Now,

(a) For $\left\{P_{x}^{1}, P_{y}^{1}\right\}$, the Jacobian matrix is of the form

$$
\begin{aligned}
& J_{T^{2}}\left(P_{x}^{1}\right)=\left(\begin{array}{cc}
1+\sqrt{1-4 c f} & -d \\
0 & 0
\end{array}\right), \\
& J_{T^{2}}\left(P_{y}^{1}\right)=\left(\begin{array}{cc}
0 & 0 \\
0 & 1+\sqrt{1-4 c f}
\end{array}\right) .
\end{aligned}
$$

with eigenvalues $\lambda_{1}=0$ and $\lambda_{2}=1+\sqrt{1-4 c f}$. Clearly, $\left\{P_{x}^{1}, P_{y}^{1}\right\}$ are saddle points.

(b) As of $\left\{P_{x}^{2}, P_{y}^{2}\right\}$, the corresponding Jacobian matrix is given by

$$
\begin{aligned}
& J_{T^{2}}\left(P_{x}^{2}\right)=\left(\begin{array}{cc}
1-\sqrt{1-4 c f} & -d \\
0 & 0
\end{array}\right), \\
& J_{T^{2}}\left(P_{y}^{2}\right)=\left(\begin{array}{cc}
0 & 0 \\
0 & 1-\sqrt{1-4 c f}
\end{array}\right) .
\end{aligned}
$$

Both with eigenvalues $\lambda_{1}=0$ and $\lambda_{2}=1-\sqrt{1-4 c f}<1$. Clearly, $\left\{P_{x}^{2}, P_{y}^{2}\right\}$ are locally asymptotically stable.

(3) Now, at the interior period-two solutions $\left\{P_{\mp}^{i}, P_{ \pm}^{i}\right\}$, the Jacobian matrices are

$$
\begin{aligned}
& J_{T^{2}}\left(P_{\mp}^{i}\right)=\left(\begin{array}{cc}
\frac{-d c+\sqrt{-c^{2}(d(3 d+2)+4 c f-1)}}{c}+1 & -d \\
-\frac{d\left(-d c+c+\sqrt{-c^{2}(d(3 d+2)+4 c f-1)}\right)}{c} & \frac{(d-1) d c-\sqrt{-c^{2}(d(3 d+2)+4 c f-1)}}{c}+1
\end{array}\right), \\
& J_{T^{2}}\left(P_{ \pm}^{i}\right)=\left(\begin{array}{cc}
\frac{-d c-\sqrt{-c^{2}(d(3 d+2)+4 c f-1)}}{c}+1 & -d \\
\frac{d\left(c(d-1)+\sqrt{-c^{2}(d(3 d+2)+4 c f-1)}\right)}{c} & \frac{\left(d^{2}-d\right) c+\sqrt{-c^{2}(d(3 d+2)+4 c f-1)}}{c}+1
\end{array}\right) .
\end{aligned}
$$

Observe that

$$
\begin{aligned}
& p=\operatorname{TrJ}_{T^{2}}\left(P_{\mp}^{i}\right)=\operatorname{TrJ}_{T^{2}}\left(P_{ \pm}^{i}\right)=2+d(d-2), \\
& q=\operatorname{DetJ}_{T^{2}}\left(P_{\mp}^{i}\right)=\operatorname{Det} \operatorname{Dr} J_{T^{2}}\left(P_{ \pm}^{i}\right)=4\left(d^{2}+c f\right) .
\end{aligned}
$$

Moreover,

$|p|>|1+q| \Leftrightarrow(d-1)^{2}-4 d^{2}>4 c f$ (condition of existence).

Consequently, the interior period-two solutions $\left\{P_{\mp}^{i}, P_{ \pm}^{i}\right\}$ are saddle points whenever they exist.

Remark 2. Observing that the interior period-two solutions $\left\{P_{\mp}^{i}, P_{+}^{i}\right\}$ exist if and only if

$$
\begin{aligned}
& \text { (1) } d<(1 / 3), \quad \text { since } \quad(d-1)^{2}-4 d^{2}>4 c f \Longrightarrow \\
& (d-1)^{2}-4 d^{2}>0 \Longrightarrow(1-3 d)(1+d)>0 .
\end{aligned}
$$

(2) There are 3 equilibrium points $E_{0}, E^{-}, E^{+}$where $E^{+}$is a saddle point.

3.3. Global Dynamics of Equation (1). The global dynamics of equation (1) is quite complicated. Thus, we provide the following three diagrams that describe all possible bifurcations produced by different values of parameters $d$ and $4 f c$.

Theorem 5. If $4 f c>1$, then the equilibrium $E_{0}$ is globally asymptotically stable (see Figure 4).

proof. First, observe that every solution of equation (1) is bounded, as for $x_{n-1} \neq 0$, 


$$
x_{n+1}=\frac{x_{n-1}^{2}}{c x_{n-1}^{2}+d x_{n}+f}=\frac{1}{c+\left(d x_{n} / x_{n-1}^{2}\right)+\left(f / x_{n-1}^{2}\right)}<\frac{1}{c},
$$

Moreover, by Theorem 5 from [19], subsequences $\left\{x_{2 n}\right\}_{n=0}^{\infty}$ and $\left\{x_{2 n+1}\right\}_{n=0}^{\infty}$ are eventually monotonic. Now, as for $4 f c>1$, there are no minimal period-two solutions, and we conclude that both $x_{2 n}$ and $x_{2 n+1}$ must converge to the unique equilibrium $\bar{x}=0$.

Theorem 6. Let $\mathscr{B}(A)$ denote the basin of attraction of the set $A$.

(1) The $x$-axis $[0,+\infty) \times\{0\}$ and the $y$-axis $\{0\} \times[0,+\infty)$ are invariant by $T^{2}$.

(2) If $4 f c=1$, then

(a) $(1 / 2 c,+\infty) \times\{0\} \subset \mathscr{B}\left(P_{x}\right)$;

(b) $\{0\} \times(1 / 2 c,+\infty) \subset \mathscr{B}\left(P_{y}\right)$;

(c) $\{0\} \times(0,1 / 2 c) \cup(0,1 / 2 c) \times\{0\} \subset \mathscr{B}\left(E_{0}\right)$.

(3) If $4 f c<1$, then
(a) $((1-\sqrt{1-4 c f}) / 2 c, \infty) \times\{0\} \subset \mathscr{B}\left(P_{x}^{2}\right)$;
(b) $\{0\} \times((1-\sqrt{1-4 c f}) / 2 c, \infty) \subset \mathscr{B}\left(P_{y}^{2}\right)$;
(c) $(0,(1-\sqrt{1-4 c f}) / \quad 2 c) \times\{0\} \cup\{0\} \times(0,(1-$ $\sqrt{1-4 c f}) / 2 c) \subset \mathscr{B}\left(E_{0}\right)$.

Proof

(1) Let $\alpha \geq 0$, then $T^{2}(\alpha, 0)=\left(\alpha^{2} /\left(c \alpha^{2}+f\right), 0\right)$ and $T^{2}(0, \alpha)=\left(0, \alpha^{2} /\left(c \alpha^{2}+f\right)\right)$ which implies that the sets $[0,+\infty) \times\{0\}$ and $\{0\} \times[0,+\infty)$ are invariant by $T^{2}$.

(2) First, recalling that every solution of equation (1) is bounded and by Theorem 5 from [19], every solution must either converge to an equilibrium or a minimal period-two solution. It follows that every solution generated by $T^{2}$ must converge to an equilibrium. Now, consider $\left\{s_{n}\right\}_{n=1}^{\infty}$ the solution with initial point $s_{1}=\left(x_{1}, 0\right) \in(1 / 2 c,+\infty) \times\{0\}$, then

$$
\begin{aligned}
& T^{2}\left(x_{n}, 0\right)=\left(x_{n+1}, 0\right)=\left(\frac{x_{n}^{2}}{c x_{n}^{2}+f}, 0\right), \\
& x_{n+1}-x_{n}=\frac{-x_{n}\left(c x_{n}^{2}-x_{n}+f\right)}{c x_{n}^{2}+f} .
\end{aligned}
$$

(a) If $4 f c=1$, one can easily show that $x_{n}$ is monotone decreasing; thus,

(i) if $x_{n}>1 / 2 c$, then $s_{n}=\left(x_{n}, 0\right) \longrightarrow(1 / 2 c, 0)$.

(ii) If $x_{n}<1 / 2 c$, then $s_{n}=\left(x_{n}, 0\right) \longrightarrow(0,0)$

(b) If $4 f c<1$, one can easily show that $x_{n}$ is monotone decreasing in $\quad(0,(1-\sqrt{1-4 f c}) / 2 c) \cup((1+$ $\sqrt{1-4 f c}) / 2 c,+\infty)$ and monotone increasing in $((1-\sqrt{1-4 f c}) / 2 c,(1+\sqrt{1-4 f c}) / 2 c)$; thus,

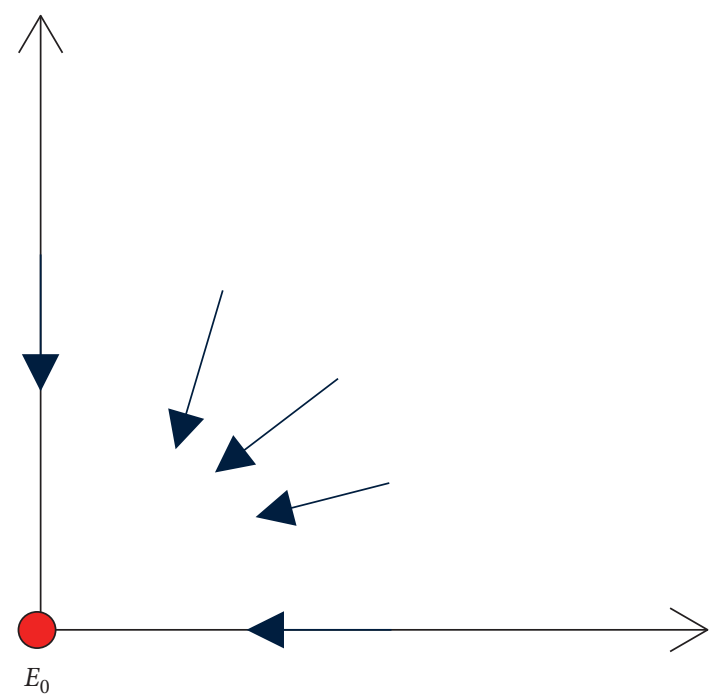

Figure 4: Visualization of Theorem 5 for $4 f c>1$.

(i) If $x_{n} \in((1-\sqrt{1-4 f c}) / 2 c,+\infty)$, then $s_{n}=\left(x_{n}, 0\right)$ $\longrightarrow((1+\sqrt{1-4 f c}) / 2 c, 0)$.

(ii) If $x_{n} \in(0,(1-\sqrt{1-4 f c}) / 2 c)$, then $s_{n}=\left(x_{n}, 0\right)$ $\longrightarrow(0,0)$.

The remaining part of the proof follows similarly by considering solutions of the form $s_{n}=\left(0, x_{n}\right)$.

Theorem 7. If $4 f c=1$, then equation (1) has the unique equilibrium point $E_{0}$ which is locally asymptotically stable and the unique minimal period-two solution $\left\{P_{x}, P_{y}\right\}=\{(1 / 2 c, 0),(0,1 / 2 c)\}$ which is nonhyperbolic of stable type.

There exist two invariant curves $\mathscr{C}_{1}$ and $\mathscr{C}_{2}$ which are graphs of strictly increasing continuous functions of the first coordinate on an interval with endpoints in $P_{x}$ and $P_{y}$, respectively. Basins of attraction of the minimal period-two solutions are

$$
\begin{aligned}
& \mathscr{B}\left(P_{x}\right)=\mathscr{C}_{1} \cup \mathscr{W}^{+}\left(\mathscr{C}_{1}\right), \\
& \mathscr{B}\left(P_{y}\right)=\mathscr{C}_{2} \cup \mathscr{W}^{-}\left(\mathscr{C}_{2}\right),
\end{aligned}
$$

while the basin of attraction of the equilibrium point $E_{0}$ is the region between curves $\mathscr{C}_{1}$ and $\mathscr{C}_{2}$, i.e.,

$$
\mathscr{B}\left(E_{0}\right)=\mathscr{W}^{-}\left(\mathscr{C}_{1}\right) \cap \mathscr{W}^{+}\left(\mathscr{C}_{2}\right) ;
$$

see Figure 5.

Proof. The Jacobian matrix of the second iterate of the map at $P_{x}$ is given by

$$
J_{T^{2}}\left(P_{x}\right)=\left(\begin{array}{cc}
1 & -d \\
0 & 0
\end{array}\right)
$$




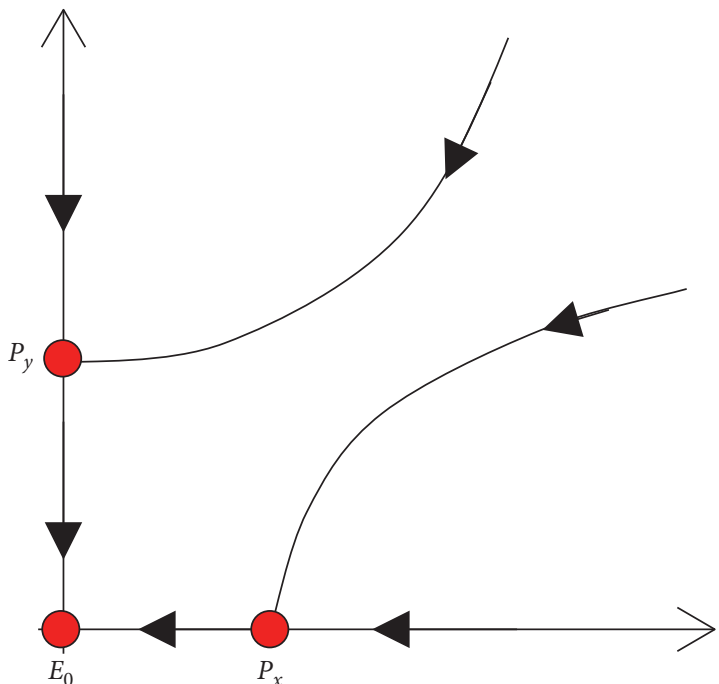

Figure 5: Visualization of global dynamics of Theorem 7 for $4 f c=1$.

with two eigenvalues $\lambda_{1}=0$ associated with the eigenvector $\left(\begin{array}{l}d \\ 1\end{array}\right)$ and $\lambda_{2}=1$ which corresponds to the eigenvector $\left(\begin{array}{l}1 \\ 0\end{array}\right)$.

Observe that the eigenvector associated with $\lambda_{1}$ is not parallel to the $x$-axis and the map $T^{2}$ is strongly competitive. It follows by Theorems 1-5 in [15] and Theorem 2 that there exists an invariant curve $\mathscr{C}_{1}$ through the point $P_{x}$ which is a subset of $\mathscr{W}^{s}\left(P_{x}\right)$. Moreover, $\mathscr{C}_{1}$ is the graph of a strictly increasing continuous that separates the first quadrant into two connected subregions: an upper one $\mathscr{W}^{-}\left(\mathscr{C}_{1}\right)$ and a lower one $\mathscr{W}^{+}\left(\mathscr{C}_{1}\right)$, where

$$
\mathscr{B}\left(P_{x}\right)=\mathscr{C}_{1} \cup \mathscr{W}^{+}\left(\mathscr{C}_{1}\right) .
$$

The Jacobian matrix of $T^{2}$ at $P_{y}$ is $J_{T^{2}}\left(P_{y}\right)=\left(\begin{array}{ll}0 & 0 \\ 0 & 1\end{array}\right)$ and it has two eigenvectors that are parallel to the coordinate axis, $\left(\begin{array}{l}1 \\ 0\end{array}\right),\left(\begin{array}{l}0 \\ 1\end{array}\right)$, corresponding to $\lambda_{1}=0$ and $\lambda_{2}=1$, respectively. By Hartman-Grobman theorem [20], we know that there exists a $C^{1}$ curve $\mathscr{C}$ through $P_{y}$ that is tangential at $P_{y}$ to the eigenspace associated with $\lambda=0$ such that $T^{2}(\mathscr{C}) \subset \mathscr{C}$.

Claim 1. The stable manifold at $P_{y}$ is a linearly strongly ordered curve in the northeast ordering, given as $\mathscr{W}_{\text {loc }}^{s}\left(P_{y}\right)=\{(t, \phi(t)): 0 \leq t \leq \delta\}$ for small enough $\delta>0$.

Proof of Claim 1. First, recall that $(0,1 / 2 c) \times(0$, $1 / 2 c) \subset \mathscr{B}\left(E_{0}\right)$. Now, let $u_{0}>0$. Since $T^{2}$ is strongly competitive, we have $T^{2}\left(P_{y}\right) \ll_{s e} T^{2}\left(u_{0}, 1 / 2 c\right)$ and that implies $T^{2}\left(u_{0}, 1 / 2 c\right) \in \operatorname{int}\left(Q_{4}\left(P_{y}\right)\right)$. Therefore, there exists a ball $B_{\varepsilon}\left(T^{2}\left(u_{0}, 1 / 2 c\right)\right)$ such that $B_{\varepsilon}\left(T^{2}\left(u_{0}, 1 / 2 c\right)\right) \subset \operatorname{int}\left(Q_{4}\left(P_{y}\right)\right)$. Since the map $T^{2}$ is continuous on $\mathbb{R}_{+}^{2}$, there exists a ball $B_{\delta_{1}}\left(\left(u_{0}, 1 / 2 c\right)\right)$ such that

$$
T^{2}\left(B_{\delta_{1}}\left(\left(u_{0}, 1 / 2 c\right)\right)\right) \subset B_{\varepsilon}\left(T^{2}\left(u_{0}, 1 / 2 c\right)\right) \subset \operatorname{int}\left(Q_{4}\left(P_{y}\right)\right),
$$

which implies $T^{2 n}(u, v) \longrightarrow(0,0)$ when $n \longrightarrow \infty$ for all points $(u, v) \in B_{\delta_{1}}\left(\left(u_{0}, 1 / 2 c\right)\right)$. It follows that $\mathscr{W}_{\text {loc }}^{s}\left(P_{y}\right) \cap$ $\operatorname{int}\left(Q_{4}\left(P_{y}\right)\right)=\varnothing$. Now, observe that $\phi^{\prime}(0)=0$ as its graph must be tangential to the horizontal eigenspace. Moreover, $\phi^{\prime \prime} \geq 0$ in a small neighborhood of $t=0$; otherwise,

$\phi^{\prime \prime} \leq 0 \Longrightarrow$ there exists $\delta>0$ such that $\phi(t)$ is decreasing in $(0, \delta) \Longrightarrow \phi^{\prime}(t) \leq 0$ in $(0, \delta)$

$\Longrightarrow \phi(t) \leq 1 / 2 c$ in $(0, \delta)$ which contradicts the fact that $\mathscr{W}_{\text {loc }}^{s}\left(P_{y}\right) \cap \operatorname{int}\left(Q_{4}\left(P_{y}\right)\right)=\varnothing$.

Therefore, for sufficiently small $\delta_{1}$, $\mathscr{W}_{\text {loc }}^{s}\left(P_{y}\right)=\left\{(t, \phi(t)): 0 \leq t \leq \delta_{1}\right\}$ is linearly ordered in the northeast ordering and as $T^{2}$ is competitive, $\mathscr{W}_{\text {loc }}^{s}\left(P_{y}\right) \cap \mathbb{R}_{+}^{2}$ can be extended to an unbounded curve (global stable manifold) $\mathscr{C}_{2}$, see $[14,15]$.

Hence, the curve $\mathscr{C}_{2}$ splits the region into two connected components, an upper subregion $\mathscr{W}^{-}\left(\mathscr{C}_{2}\right)$ and a lower subregion $\mathscr{W}^{+}\left(\mathscr{C}_{2}\right)$.

Clearly, $\mathscr{B}\left(P_{y}\right)=\mathscr{C}_{2} \cup \mathscr{W}^{-}\left(\mathscr{C}_{2}\right)$, and finally the basin of attraction of the zero equilibrium $E_{0}$ is

$$
\mathscr{B}\left(E_{0}\right)=\mathscr{W}^{+}\left(\mathscr{C}_{2}\right) \cap \mathscr{W}^{-}\left(\mathscr{C}_{1}\right) .
$$

Theorem 8. If $(d \geq 1$ and $4 f c<1)$ or $\left(d<1\right.$ and $(d-1)^{2}<$ $4 f c<1$ ), then equation (1) has the unique equilibrium point $E_{0}$ which is locally asymptotically stable and two minimal period-two solutions $\left\{P_{x}^{1}, P_{y}^{1}\right\}$ which is a saddle point and $\left\{P_{x}^{2}, P_{y}^{2}\right\}$ which is locally asymptotically stable.

There exist global stable manifolds $\mathscr{W}^{s}\left(P_{x}^{1}\right)$ and $\mathscr{W}^{s}\left(P_{y}^{1}\right)$ which are basins of attractions of $\left\{P_{x}^{1}, P_{y}^{1}\right\}$ and the global unstable manifolds have the following form:

$$
\begin{aligned}
& \mathscr{W}^{u}\left(P_{x}^{1}\right)=\{(x, 0): x \in A\}, \\
& \mathscr{W}^{u}\left(P_{y}^{1}\right)=\{(0, y): y \in A\},
\end{aligned}
$$

where $A=(0,(1+\sqrt{1-4 c f}) / 2 c) \backslash\{(1-\sqrt{1-4 c f}) / 2 c\}$.

The basin $\mathscr{B}\left(E_{0}\right)=(0,0)$ is the region between the global stable sets:

$$
\mathscr{B}\left(E_{0}\right)=\mathscr{W}^{-}\left(P_{x}^{1}\right) \cap \mathscr{W}^{+}\left(P_{y}^{1}\right) .
$$

The basin of the minimal period-two solutions $\left\{P_{x}^{2}, P_{y}^{2}\right\}$ is

$$
\begin{aligned}
& \mathscr{B}\left(P_{x}^{2}\right)=\mathscr{W}^{+}\left(P_{x}^{1}\right), \\
& \mathscr{B}\left(P_{y}^{2}\right)=\mathscr{W}^{-}\left(P_{y}^{1}\right) ;
\end{aligned}
$$

see Figure 6.

Proof. Recall that

$$
J_{T^{2}}\left(P_{x}^{1}\right)=\left(\begin{array}{cc}
1+\sqrt{1-4 c f} & -d \\
0 & 0
\end{array}\right),
$$

with eigenvalues $\lambda_{1}=0$ with eigenvector $\left(\begin{array}{c}d /(\sqrt{1-4 f c}+1) \\ 1\end{array}\right)$ and $\lambda_{2}=\sqrt{1-4 \mathrm{fc}}+1$ associated with 


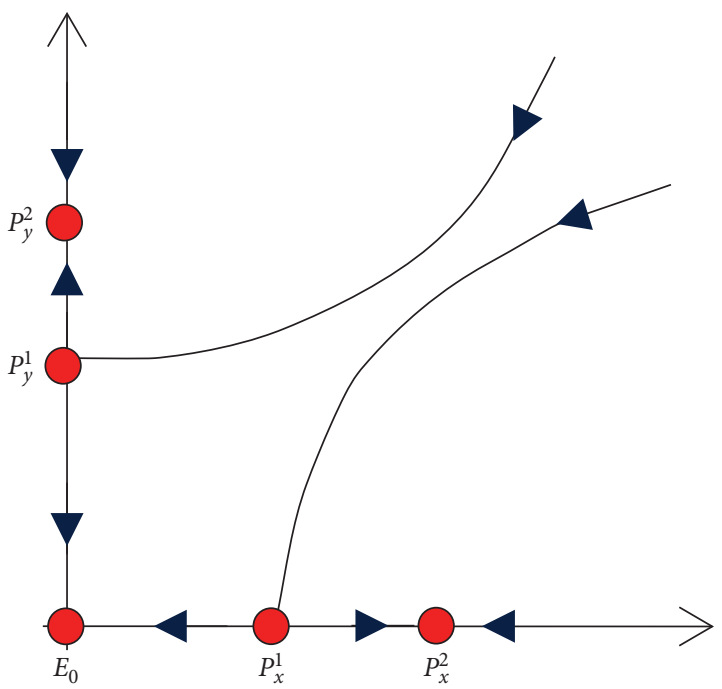

Figure 6: Different manifolds of Theorem 8.

the eigenvector $\left(\begin{array}{l}1 \\ 0\end{array}\right)$. Thus, there exists a local stable manifold at $P_{x}^{1}$ that is linearly strongly ordered in the northeast ordering with $P_{x}^{1}$ as an endpoint. As $T^{2}$ is competitive, the local stable manifold can be extended to a curve $\mathscr{W}^{s}\left(P_{x}^{1}\right)$ which separates the region into two connected components $\mathscr{W}^{+}\left(P_{x}^{1}\right)$ and $\mathscr{W}^{-}\left(P_{x}^{1}\right)$. On the other hand,

$$
J_{T^{2}}\left(P_{y}^{1}\right)=\left(\begin{array}{cc}
0 & 0 \\
0 & 1+\sqrt{1-4 c f}
\end{array}\right),
$$

with eigenvalues $\lambda_{1}=0$ with eigenvector $\left(\begin{array}{l}1 \\ 0\end{array}\right)$ and $\lambda_{2}=$ $\sqrt{1-4 \mathrm{fc}}+1$ associated with the eigenvector $\left(\begin{array}{l}0 \\ 1\end{array}\right)$.

By Theorem 6, we know that $(0,(1-\sqrt{1-4 f c}) / 2 c) \times$ $(0,(1-\sqrt{1-4 f c}) / 2 c) \subset \mathscr{B}\left(E_{0}\right)$; thus, we know that the local stable manifold at $P_{y}^{1}$ is tangential to the horizontal eigenspace but cannot enter the box $(0,(1-\sqrt{1-4 f c}) / 2 c)$ $\times(0,(1-\sqrt{1-4 f c}) / 2 c)$. We conclude that the local stable manifold at $P_{y}^{1}$ is a linearly strongly ordered curve (in the northeast ordering) with $P_{y}^{1}$ as an endpoint. Similarly, we conclude its extension to a global stable manifold $\mathscr{W}^{s}\left(P_{y}^{1}\right)$ which separates the region into two connected components $\mathscr{W}^{+}\left(P_{y}^{1}\right)$ and $\mathscr{W}^{-}\left(P_{y}^{1}\right)$.

Finally, by the uniqueness of the stable manifold of the saddle point $P_{x}^{1}$, we know that no solution in $\mathscr{W}^{+}\left(P_{x}^{1}\right)$ will converge to $P_{x}^{1}$. Furthermore, all solutions are bounded and we know that by monotonicity of the map $T$ every solution must converge to an equilibrium. It follows that $\mathscr{B}\left(P_{x}^{2}\right)=$ $\mathscr{W}^{+}\left(P_{x}^{1}\right)$ and analogously $\mathscr{B}\left(P_{y}^{2}\right)=\mathscr{W}^{+}\left(P_{y}^{1}\right)$.

Theorem 9. If $d<1$ and $(d-1)^{2}=4 f c<1$, then equation (1) has two equilibrium solutions:

(i) $E_{0}$ is locally asymptotically stable (ii) $E^{*}=((1-d) / 2 c,(1-d) / 2 c)$ is a nonhyperbolic of unstable type, and two minimal period-two solutions:

(iii) $\left\{P_{x}^{1}, P_{y}^{1}\right\}=\{((1-\sqrt{1-4 f c}) / 2 c, 0)$, $\sqrt{1-4 f c}) / 2 c)\}$ are saddle points,

$(0,(1-$

(iv) $\left\{P_{x}^{2}, P_{y}^{2}\right\}=\{((1+\sqrt{1-4 f c}) / 2 c, 0),(0,(1+$ $\sqrt{1-4 f c}) / 2 c)\}$ are locally asymptotically stable.

Then,

(i) there exist global stable manifolds $\mathscr{W}^{s}\left(P_{x}^{1}\right)$ and $\mathscr{W}^{s}\left(P_{y}^{1}\right)$ which are the basins of attraction of the periodic solutions $\left\{P_{x}^{1}, P_{y}^{1}\right\}$ and which are tangential at the equilibrium point $E^{*}$.

(ii) There exists a global stable manifold $\mathscr{W}^{s}\left(E^{*}\right)$ contained in $\mathbb{Q}_{1}\left(E^{*}\right)$ which is the basin of attraction of the equilibrium $E^{*}$;

The basin of attraction of the equilibrium point $E_{0}$ is given by

$$
\mathscr{B}\left(E_{0}\right)=\mathscr{W}^{-}\left(P_{x}^{1}\right) \cap \mathscr{W}^{+}\left(P_{y}^{1}\right) .
$$

The basins of attraction of $P_{x}^{2}$ and $P_{y}^{2}$ are given by

$$
\begin{aligned}
& \mathscr{B}\left(P_{x}^{2}\right)=\mathscr{W}^{+}\left(S_{x}\right), \text { where } S_{x}=\mathscr{W}^{s}\left(P_{x}^{1}\right) \cup \mathscr{W}^{s}\left(E^{*}\right), \\
& \mathscr{B}\left(P_{y}^{2}\right)=\mathscr{W}^{-}\left(S_{y}\right), \text { where } S_{y}=\mathscr{W}^{s}\left(P_{y}^{1}\right) \cup \mathscr{W}^{s}\left(E^{*}\right) ;
\end{aligned}
$$

see Figure 7.

Proof. The existence and orientation of the global stable manifold at $P_{x}^{1}$ can be determined as in Theorem 8; however, in general, this information can be determined by studying the curvature of the local curves given by

$\mathscr{W}_{\text {loc }}^{s}\left(P_{y}^{1}\right)=\left\{t, \phi_{1}(t): 0 \leq t \leq \delta_{1}\right\}$ and $\mathscr{W}_{\text {loc }}^{s}\left(P_{x}^{1}\right)=\left\{\left(\phi_{2}\right.\right.$ $\left.(t), t): 0 \leq t \leq \delta_{2}\right\}$ for $\delta_{1}$ and $\delta_{2}$ small enough, where if $f(x, y)$ and $g(x, y)$ are the coordinate functions of $T^{2}$, then

$$
\begin{aligned}
\phi_{1}\left(f\left(t, \phi_{1}(t)\right)\right) & =g\left(t, \phi_{1}(t)\right), \\
\phi_{1}(0) & =\frac{1-\sqrt{1-4 f c}}{2 c}, \\
\phi_{1}^{\prime}(0) & =0, \\
\phi_{2}\left(g\left(\phi_{2}(t), t\right)\right) & =f\left(\phi_{2}(t), t\right), \\
\phi_{2}(0) & =\frac{1-\sqrt{1-4 f c}}{2 c}, \\
\phi_{2}^{\prime}(0) & =0 .
\end{aligned}
$$

This is useful when the local curve has a tangent parallel to the axis at the fixed point which is the case here for $p_{y}^{1}$. get

By differentiating both sides of the equation above, we 


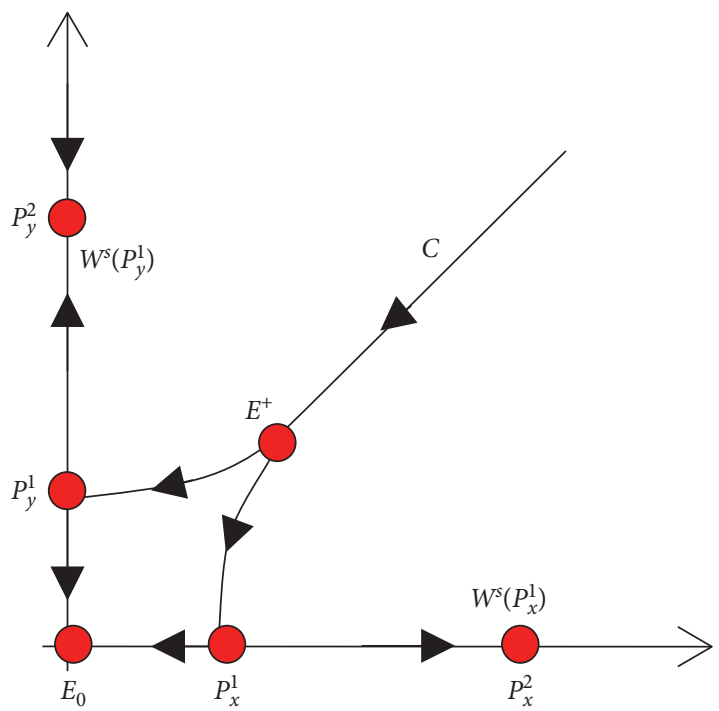

Figure 7: Global manifolds and basins of Theorem 9.

$$
\begin{aligned}
\phi_{1}^{\prime \prime}(0) & =\frac{g_{x x}\left(0, \phi_{1}(0)\right)}{\left(f_{x}\left(0, \phi_{1}(0)\right)\right)^{2}-g_{y}\left(0, \phi_{1}(0)\right)} \\
& =\frac{d(1-\sqrt{1-4 f c})}{f(d+2 c f-d \sqrt{1-4 f c})}>0,
\end{aligned}
$$

which confirms the argument used in Theorem 8. We conclude the existence of curves $\mathscr{C}_{1}$ and $\mathscr{C}_{2}$ (global stable manifolds) which are graphs of continuous, strictly increasing functions. Furthermore, the curves cannot intersect the interior of the sets $Q_{2}\left(E^{*}\right) \cap \mathbb{R}^{2}$ and $Q_{4}\left(E^{*}\right) \cap \mathbb{R}^{2}$, as the monotonicity of $T^{2}$ forces the latter sets to be invariant. Thus, $T^{-2 n}(P) \longrightarrow E^{*}$ for all $P \in \mathscr{C}_{l}, l=1,2$, therefore $\mathscr{C}_{1}$ and $\mathscr{C}_{2}$ are also center manifolds of $E^{*}$.

On the other hand, by letting $T^{2}(x, y)=f(x, y)$, $g(x, y)$, the center manifold $\phi(x)$ must satisfy [21]

$$
\phi(f(x, \phi(x)))=g(x, \phi(x)) .
$$

By using a Taylor expansion substitution, the center manifold can be approximated by

$$
\begin{aligned}
\phi(x)= & x-\frac{c(x-((1-d) / 2 c))^{2}}{d+2}-\frac{2 c^{2}(x-((1-d) / 2 c))^{3}}{(d+2)^{3}} \\
& +O\left(\left|x-\frac{1-d}{2 c}\right|^{4}\right) .
\end{aligned}
$$

The dynamic on the center manifold $\phi(x)$ is given by the reduced difference equation $u_{n+1}=f\left(u_{n}, \phi\left(u_{n}\right)\right)$ which has the following asymptotic representation:

$$
\begin{aligned}
u_{n+1}= & \frac{1-d}{2 c}+\left(u_{n}-\frac{1-d}{2 c}\right)-\frac{2 c\left(u_{n}-(1-d) / 2 c\right)^{2}}{d+2} \\
& +\frac{2 c^{2} d\left(u_{n}-(1-d) / 2 c\right)^{3}}{(d+2)^{3}}-\cdots+O\left(\left|u_{n}-\frac{1-d}{2 c}\right|^{5}\right) .
\end{aligned}
$$

Since $\bar{u}=(1-d) / 2 c$ is a semistable fixed point for the latter scalar difference equation, it follows that $E^{*}$ is a semistable fixed point for $T^{2}$; furthermore, the coefficient of the lowest nonlinear term in the reduced map is negative; thus, by [22], the local basin of attraction of the equilibrium $E^{*}$ is a one-dimensional curve. We conclude that there is a unique center manifold curve $\mathfrak{U}$ which satisfies $T^{2}(\mathfrak{U}) \subset \mathfrak{U}$. Moreover, $\mathfrak{U}$ is tangential to the eigenspace associated with $\lambda=1$, namely, $\operatorname{Span}\left\{\left(\begin{array}{l}1 \\ 1\end{array}\right)\right\}$. It follows that $\mathcal{U}$ is contained in $Q_{1}\left(E^{*}\right)$ and is linearly ordered in the northeast ordering and therefore can be extended to an unbounded curve $\mathscr{C}$.

Now, for every point $q \in \mathscr{W}^{-}\left(P_{x}^{1}\right) \cap \mathscr{W}^{+}\left(P_{y}^{1}\right)$, there exist $q_{x} \in \mathscr{W}^{s}\left(P_{x}^{1}\right)$ and $q_{y} \in \mathscr{W}^{s}\left(P_{y}^{1}\right)$ such that

$q_{y} \unlhd_{s e} q \unlhd_{s e} q_{x}$ which implies that $T^{2 n}\left(q_{y}\right) \preceq_{s e} T^{2 n}(q) \preceq s_{s e}$ $T^{2 n}\left(q_{x}\right)$, but we know that

$$
\begin{aligned}
& T^{2 n}\left(q_{y}\right) \longrightarrow P_{y}^{1}, \\
& T^{2 n}\left(q_{x}\right) \longrightarrow P_{x}^{1} .
\end{aligned}
$$

Consequently, there exists $N$ such that

$$
P_{y}^{1} \varsigma_{s e} T^{2 N}(q){ }_{s e} P_{x}^{1} .
$$

It follows by Theorem 6 that $q \in \mathscr{B}\left(E_{0}\right)$. As of the basins of attractions of $P_{x}^{2}$ and $P_{y}^{2}$, the proof is analogous to the one given in Theorem 8 .

Theorem 10. If $d<1,(d-1)^{2}-4 d^{2}<4 f c<(d-1)^{2}$, then equation (1) has three equilibrium points $E_{0}, E^{-}, E^{+}$, which are, respectively, locally asymptotically stable, a repeller and a saddle point and two minimal period-two solutions:

(i) $\left\{P_{x}^{1}, P_{y}^{1}\right\}$ are saddle points

(ii) $\left\{P_{x}^{2}, P_{y}^{2}\right\}$ are locally asymptotically stable

Then,

(i) There exist global stable manifolds $\mathscr{W}^{s}\left(P_{x}^{1}\right)$ (the basin of attraction of $P_{x}^{1}$ ) and $\mathscr{W}^{s}\left(P_{y}^{1}\right)$ (the basin of attraction of $P_{x}^{2}$ ) which are tangential at the equilibrium point $E^{-}$.

(ii) There exists a global stable manifold $\mathscr{W}^{s}\left(E^{+}\right)$which is an unbounded curve that is a graph of an increasing function contained in $Q_{1}\left(E^{+}\right) \cup Q_{3}\left(E^{+}\right)$ with an endpoint at $E^{-}$and is the basin of attraction of the equilibrium $E^{+}$. 
(iii) There exist a global unstable manifold $\mathscr{W}^{u}\left(E^{+}\right)$ which is a graph of a decreasing function contained in $\mathbb{Q}_{2}\left(E^{+}\right) \cup \mathbb{Q}_{4}\left(E^{+}\right)$with endpoints $P_{x}^{2}$ and $P_{y}^{2}$.

(iv) The basin of attraction of $E_{0}$ is

$$
\mathscr{B}\left(E_{0}\right)=\mathscr{W}^{-}\left(P_{x}^{1}\right) \cap \mathscr{W}^{+}\left(P_{y}^{1}\right) .
$$

(v) The basins of attraction of $P_{x}^{2}$ and $P_{y}^{2}$ are given by

$$
\begin{aligned}
& \mathscr{B}\left(P_{x}^{2}\right)=\mathscr{W}^{+}\left(S_{x}\right), \text { where } S_{x}=\mathscr{W}^{s}\left(P_{x}^{1}\right) \cup \mathscr{W}^{s}\left(E^{+}\right), \\
& \mathscr{B}\left(P_{y}^{2}\right)=\mathscr{W}^{-}\left(S_{y}\right), \text { where } S_{y}=\mathscr{W}^{s}\left(P_{y}^{1}\right) \cup \mathscr{W}^{s}\left(E^{+}\right) ;
\end{aligned}
$$

see Figure 8.

Proof. He the existence of $\mathscr{W}^{s}\left(P_{x}^{1}\right)$ and $\mathscr{W}^{s}\left(P_{y}^{1}\right)$ as well as the basin of attraction of $E_{0}$ follows from Theorem 9 . The existence of the stable $\mathscr{W}^{s}\left(E^{+}\right)$and the unstable manifold $\mathscr{W}^{u}\left(E^{+}\right)$follows from Theorems 1-5 in [15] and Theorem 2. The basins of attraction of $P_{x}^{2}$ and $P_{y}^{2}$ were discussed in Theorem 8.

Theorem 11. If $d<1$ and $0<(d-1)^{2}-4 d^{2}=4 f c$, then equation (1) has three equilibrium points $E_{0}, E^{-}, E^{+}$, where

(i) $E_{0}$ is locally asymptotically stable

(ii) $E^{-}=((1-3 d) / 2 c,(1-3 d) / 2 c)$ is a repeller

(iii) $E^{+}=((1+d) / 2 c,(1+d) / 2 c)$ is a nonhyperbolic point of stable type, and two minimal period-two solutions:

(iv) $\left\{P_{x}^{1}, P_{y}^{1}\right\}$ is a saddle point

(v) $\left\{P_{x}^{2}, P_{y}^{2}\right\}$ is locally asymptotically stable

Then,

(i) There exist global stable manifolds $\mathscr{W}^{s}\left(P_{x}^{1}\right)$ and $\mathscr{W}^{s}\left(P_{y}^{1}\right)$ which are the basins of attraction of the periodic solutions $\left\{P_{x}^{1}, P_{y}^{1}\right\}$ and which are tangential at the equilibrium point $E^{-}$.

(ii) There exists a global stable manifold $\mathscr{W}^{s}\left(E^{+}\right)$which is an unbounded curve and the graph of an increasing function contained in $\mathbb{Q}_{1}\left(E^{+}\right) \cup Q_{3}\left(E^{+}\right)$with an endpoint at $E^{-}$and $\mathscr{W}^{s}\left(E^{+}\right)$is the basin of attraction of the equilibrium $E^{+}$.

(iii) The basin of attraction of equilibrium point $E_{0}$ is the region between those stable manifolds, i.e.,

$$
\mathscr{B}\left(E_{0}\right)=\mathscr{W}^{-}\left(P_{x}^{1}\right) \cap \mathscr{W}^{+}\left(P_{y}^{1}\right) .
$$

(iv) The basins of attraction of $P_{x}^{2}$ and $P_{y}^{2}$ are given by

$$
\begin{aligned}
& \mathscr{B}\left(P_{x}^{2}\right)=\mathscr{W}^{+}\left(S_{x}\right), \text { where } S_{x}=\mathscr{W}^{s}\left(P_{x}^{1}\right) \cup \mathscr{W}^{s}\left(E^{+}\right), \\
& \mathscr{B}\left(P_{y}^{2}\right)=\mathscr{W}^{-}\left(S_{y}\right), \text { where } S_{y}=\mathscr{W}^{s}\left(P_{y}^{1}\right) \cup \mathscr{W}^{s}\left(E^{+}\right) ;
\end{aligned}
$$

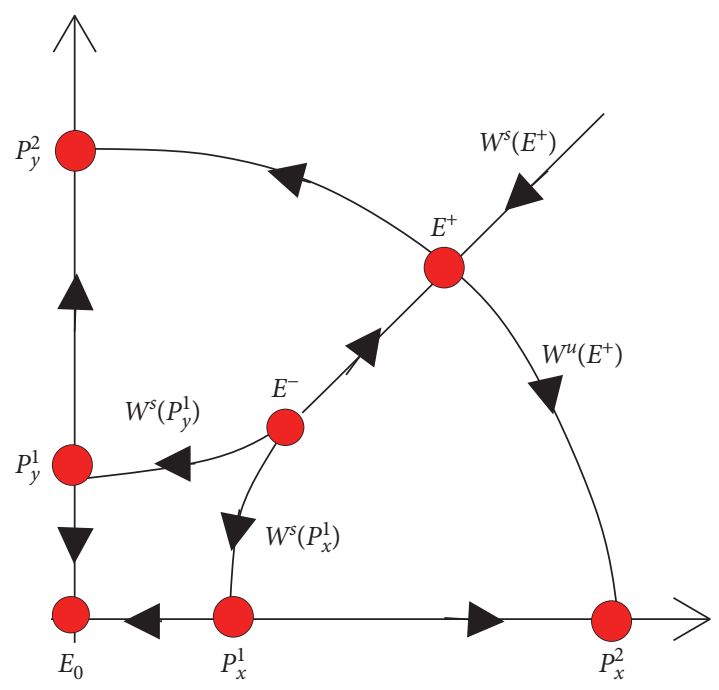

Figure 8: All invariant manifolds of Theorem 10.

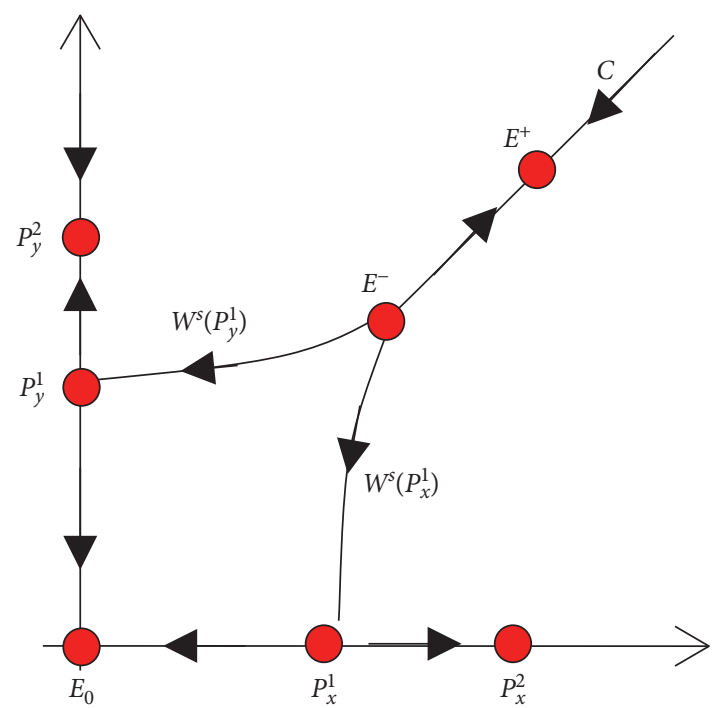

FIGURE 9: Visual representation of global dynamics of Theorem 11.

see Figure 9.

Proof. The existence and orientation of the global stable manifold $\mathscr{W}^{s}\left(E^{+}\right)$follows from Theorems $1-5$ in [15] and Theorem 2. The remaining of the proof is analogous to the discussions in Theorems 8 and 9 .

Theorem 12. If $d<1$ and $4 f c<(d-1)^{2}-4 f c$, then equation (1) has three equilibrium points $E_{0}, E_{-}, E_{+}$which are, respectively, locally asymptotically stable, a repeller and locally asymptotically stable, and has three minimal period-two solutions such that

(i) $\left\{P_{x}^{1}, P_{y}^{1}\right\}$ are saddle points,

(ii) $\left\{P_{x}^{2}, P_{y}^{2}\right\}$ are locally asymptotically stable,

(iii) $\left\{P_{\mp}^{i}, P_{+}^{i}\right\}$ are saddle points.

(iv) There exist global stable manifolds $\mathscr{W}^{s}\left(P_{x}^{1}\right)$ and $\mathscr{W}^{s}\left(P_{y}^{1}\right)$ which are the basins of attraction of the 
periodic solutions $\left\{P_{x}^{1}, P_{y}^{1}\right\}$ and which are tangential at the equilibrium point $E^{-}$.

(v) The basin of attraction of equilibrium point $E_{0}$ is the region between those stable manifolds, i.e.,

$$
\mathscr{B}\left(E_{0}\right)=\mathscr{W}^{-}\left(P_{x}^{1}\right) \cap \mathscr{W}^{+}\left(P_{y}^{1}\right) .
$$

(vi) There exists a global stable manifold $\mathscr{W}^{s}\left(P_{\mp}^{i}\right)$ which is an unbounded curve and the graph of an increasing function contained in $Q_{1}\left(P_{\mp}^{i}\right) \cup Q_{3}\left(P_{\mp}^{i}\right)$ with an endpoint at $E^{-} . \mathscr{W}^{s}\left(P_{\mp}^{i}\right)$ is the basin of attraction of the equilibrium $P_{\mp}^{i}$.

(vii) There exists a global stable manifold $\mathscr{W}^{s}\left(P^{i}{ }_{+}\right)$ which is an unbounded curve and the graph of an increasing function contained in $\mathbb{Q}_{1}\left(P_{ \pm}^{i}\right) \cup$ $Q_{3}\left(P_{+}^{i}\right)$ with an endpoint at $E^{-} . \mathscr{W}^{s}\left(P^{i}\right)$ is the basin of attraction of the equilibrium $P^{i}{ }_{ \pm}$.

(viii) The basin of attraction of the equilibrium point $E_{+}$is the region between those stable manifolds, i.e.,

$$
\mathscr{B}\left(E^{+}\right)=\mathscr{W}^{-}\left(P_{ \pm}^{i}\right) \cap \mathscr{W}^{+}\left(P_{\mp}^{i}\right) .
$$

(ix) There exist a global unstable manifold $\mathscr{W}^{u}\left(P_{\mp}^{i}\right)$ which is the graph of a decreasing function contained in $\mathbb{Q}_{2}\left(P_{\mp}^{i}\right) \cup \mathcal{Q}_{4}\left(P_{\mp}^{i}\right)$ with endpoints $P_{y}^{2}$ and $E^{+}$.

(x) There exist a global unstable manifold $\mathscr{W}^{u}\left(P^{i}\right)$ which is the graph of a decreasing function contained in $Q_{2}\left(P_{ \pm}^{i}\right) \cup Q_{4}\left(P_{ \pm}^{i}\right)$ with endpoints $P_{x}^{2}$ and $E^{+}$.

(xi) The basins of attraction of $P_{x}^{2}$ and $P_{y}^{2}$ are given by

$$
\begin{aligned}
& \mathscr{B}\left(P_{x}^{2}\right)=\mathscr{W}^{+}\left(S_{x}\right), \text { where } S_{x}=\mathscr{W}^{s}\left(P_{x}^{1}\right) \cup \mathscr{W}^{s}\left(P^{i}{ }_{ \pm}\right), \\
& \mathscr{B}\left(P_{y}^{2}\right)=\mathscr{W}^{-}\left(S_{y}\right), \text { where } S_{y}=\mathscr{W}^{s}\left(P_{y}^{1}\right) \cup \mathscr{W}^{s}\left(P_{ \pm}^{i}\right)
\end{aligned}
$$

see Figure 10.

proof. The existence and orientation of the stable manifold $\mathscr{W}^{s}\left(P_{\mp}^{i}\right)$ follows from Theorems $1-5$ in [15] and Theorem 2. Moreover, $\mathscr{W}^{s}\left(P_{\mp}^{i}\right)$ cannot intersect another manifold or the boundary of the region at any point as the latter sets are invariant. Thus, it must have an endpoint at $E^{-}$. Similarly, the existence and orientation of the unstable manifold $\mathscr{W}^{u}\left(P_{\mp}^{i}\right)$ are given by Theorem 5 in [15]. On the other hand, $\mathscr{W}^{u}\left(P_{\mp}^{i}\right) \cap\left[P_{\mp}^{i}, E^{+}\right] \neq \varnothing$ and $\left[\left(P_{\mp}^{i}, E^{+}\right]\right.$is invariant. Thus, $\mathscr{W}^{u}\left(P_{\mp}^{i}\right)$ cannot leave the latter set and must end at $E^{+}$. Analogous arguments and conclusions also hold for $\mathscr{W}^{s}\left(P^{i}{ }_{ \pm}\right)$and $\mathscr{W}^{u}\left(P^{i}{ }_{ \pm}\right)$. In addition, we know that for $p_{\mp} \in \mathscr{W}^{s}\left(P_{\mp}^{i}\right)$ and $p_{ \pm} \in \mathscr{W}^{s}\left(\dot{P}_{ \pm}^{i}\right)$,

$$
\begin{aligned}
& T^{2 n}\left(p_{\mp}\right) \longrightarrow P_{\mp}^{i}, \\
& T^{2 n}\left(p_{ \pm}\right) \longrightarrow P_{ \pm}^{i} .
\end{aligned}
$$

Furthermore, for all $p \in \mathscr{W}^{-}\left(P^{i}\right) \cap \mathscr{W}^{+}\left(P_{\mp}^{i}\right)$, there exist $p_{\mp} \in \mathscr{W}^{s}\left(P_{\mp}^{i}\right)$ and. $p_{ \pm} \in \mathscr{W}^{s}\left(P_{ \pm}^{i}\right)$ such that

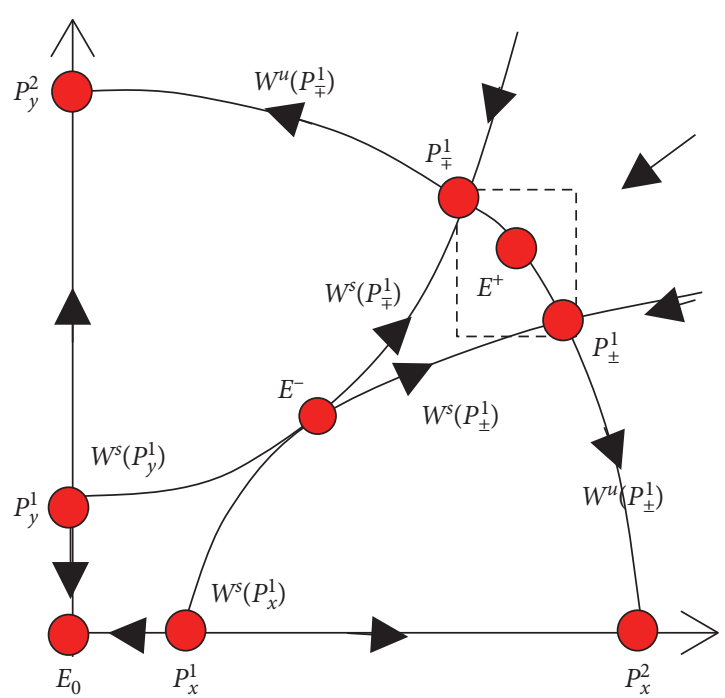

FIgURE 10: The visual illustration of Theorem 12.

$$
\begin{array}{r}
p_{\mp} \preceq_{s e} p \preceq_{s e} p_{ \pm} \Longrightarrow T^{2 n}\left(p_{\mp}\right) \preceq_{s e} T^{2 n}(p) \preceq_{s e} T^{2 n}\left(p_{ \pm}\right), \\
\text {for all } n \geq 0 .
\end{array}
$$

It follows that there exists $N>0$ such that $T^{2 N}(p)=q \in\left[P_{\mp}^{i}, P_{ \pm}^{i}\right]$. Thus, there exist $q_{\mp} \in \mathscr{W}^{u}\left(P_{\mp}^{i}\right)$ and $q_{ \pm} \in \mathscr{W}^{u}\left(P_{ \pm}^{i}\right)$ such that

$$
q_{\mp} \preceq_{s e} q \unlhd_{s e} q_{ \pm} \Longrightarrow T^{2 n}\left(q_{\mp}\right) \unlhd_{s e} T^{2 n}(q) \unlhd_{s e} T^{2 n}\left(q_{ \pm}\right), \quad \text { for all } n \geq 0,
$$

where

$$
\begin{aligned}
& T^{2 n}\left(q_{\mp}\right) \longrightarrow E^{+}, \\
& T^{2 n}\left(q_{ \pm}\right) \longrightarrow E^{+},
\end{aligned}
$$

which implies that $T^{2 n}(q) \longrightarrow E^{+} \longrightarrow T^{2 n}(p) \longrightarrow E^{+}$. We conclude that

$$
\mathscr{B}\left(E^{+}\right)=\mathscr{W}^{-}\left(P_{ \pm}^{i}\right) \cap \mathscr{W}^{+}\left(P_{\mp}^{i}\right) .
$$

As of $\mathscr{W}^{s}\left(P_{x}^{1}\right), \mathscr{W}^{s}\left(P_{y}^{1}\right), \mathscr{B}\left(E_{0}\right), \mathscr{B}\left(P_{x}^{2}\right)$ and $\mathscr{B}\left(P_{y}^{2}\right)$, the proof is analogous to the discussion in Theorems 8 and 9.

\section{Data Availability}

No data were used to support the study.

\section{Conflicts of Interest}

The authors declare that they have no conflicts of interest.

\section{References}

[1] A. M. Amleh, E. Camouzis, and G. Ladas, "On the dynamics of a rational difference equation, part I," International Journal of Difference Equations, vol. 3, pp. 1-35, 2008. 
[2] A. M. Amleh, E. Camouzis, and G. Ladas, "On the dynamics of a rational difference equation, part II," International Journal of Difference Equations, vol. 3, pp. 195-225, 2008.

[3] E. Bertrand and M. R. S. Kulenović, "Global dynamic scenarios for competitive maps in the plane," Advances in Difference Equations, vol. 291, no. 1, p. 28, 2018.

[4] M. Garić-Demirović, M. R. S. Kulenović, and M. Nurkanović, "Global dynamics of certain homogeneous second-order quadratic fractional difference equation," The Scientific World Journal, vol. 2013, p. 10, 2013.

[5] S. Jašarević- Hrustić, M. R. S. Kulenović, and M. Nurkanović, "Global dynamics and bifurcations of certain second order rational difference equation with quadratic terms," Qualitative Theory of Dynamical Systems, vol. 15, no. 1, pp. 283-307, 2016.

[6] S. Kalabušić, M. R. S. Kulenović, and M. Mehuljić, "Global period-doubling bifurcation of quadratic fractional second order difference equation," Discrete Dynamics in Nature and Society, vol. 2014, Article ID 920410, 13 pages, 2014.

[7] M. DiPippo, Global dynamics of some quadratic difference equations, Ph.D. thesis, University of Rhode Island, Kingston, RI, USA, 2016.

[8] M. R. S. Kulenović, S. Moranjkić, and M. Nurkanović, "Global dynamics and bifurcation of a perturbed sigmoid BevertonHolt difference equation," Mathematical Methods in the Applied Sciences, vol. 39, no. 10, pp. 2696-2715, 2016.

[9] C. Tunç, "A note on boundedness of solutions to a class of non-autonomous differential equations of second order," Applicable Analysis and Discrete Mathematics, vol. 4, no. 2, pp. 361-372, 2010.

[10] C. Tunç, "Uniformly stability and boundedness of solutions of second order nonlinear delay differential equations," Applied and Computational Mathematics, vol. 10, no. 3, pp. 449-462, 2011.

[11] M. Hirsch and H. Smith, Monotone Dynamical Systems, Handbook of Differential Equations: Ordinary Differential Equations, Vol. 2, Elsevier, Amsterdam, Netherlands, 2005.

[12] M. Hirsch and H. L. Smith, "Monotone maps: a review," Journal of Difference Equations and Applications, vol. 11, pp. 379-398, 2005.

[13] H. L. Smith, "Planar competitive and cooperative difference equations," Journal of Difference Equations and Applications, vol. 3, no. 5-6, pp. 335-357, 1998.

[14] M. R. S. Kulenović and O. Merino, "Global bifurcations for competitive system in the plane," Discrete and Continuous Dynamical Systems-Series B, vol. 12, no. 1, pp. 133-149, 2009.

[15] M. R. S. Kulenović and O. Merino, "Invariant manifolds for competitive discrete systems in the plane," International Journal of Bifurcation and Chaos, vol. 20, no. 8, pp. 2471-2486, 2010.

[16] M. R. S. Kulenović and O. Merino, "Invariant curves for planar competitive and cooperative maps," Journal of Difference Equations and Applications, vol. 24, no. 6, pp. 898-915, 2018.

[17] D. Burgić, S. Kalabušić, and M. R. S. Kulenović, "Non-hyperbolic dynamics for competitive systems in the plane and global period-doubling bifurcations," Advances in Dynamical Systems and Applications, vol. 3, pp. 229-249, 2008.

[18] M. R. S. Kulenović, E. Pilav, and E. Silić, "Local dynamics and global attractivity of a certain second order quadratic fractional difference equation," Advances in Difference Equations, vol. 68, no. 1, p. 32p, 2014.

[19] E. Camouzis and G. Ladas, "When does local asymptotic stability imply global attractivity in rational equations?"
Journal of Difference Equations and Applications, vol. 12, no. 8, pp. 863-885, 2006.

[20] P. Hartman, Differential Equations, Vol. 38, SIAM: Classics in Applied Mathematics, Philadelphia, PA, USA, 1982.

[21] S. Elaydi, Discrete Chaos, Chapman \& Hall/CRC, Boca Raton, FL, USA, 2000.

[22] W. T. Jamieson, Planar difference equations: asymptotic behavior of solutions and 1-1 resonant points, Ph.D. thesis, University of Rhode Island, Kingston, RI, USA, 2015. 